\title{
The Future of Spectroscopy
}

\author{
M. Darby Dyar \\ Dept. of Astronomy \\ Mount Holyoke College \\ Senior Scientist \\ Planetary Science Institute
}

The Holy Grail: Accurate Mineralogy Derived from Spectroscopy 


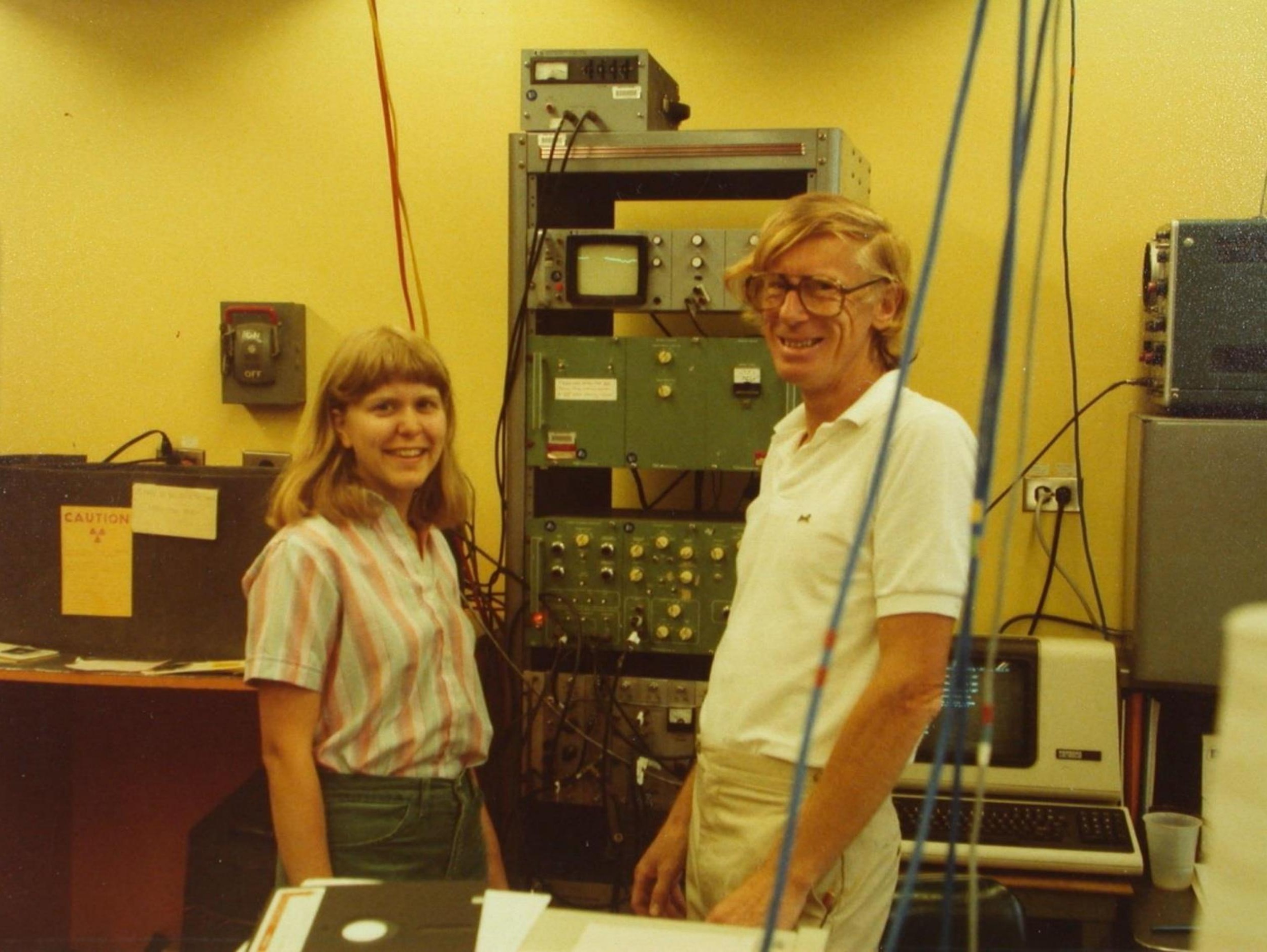




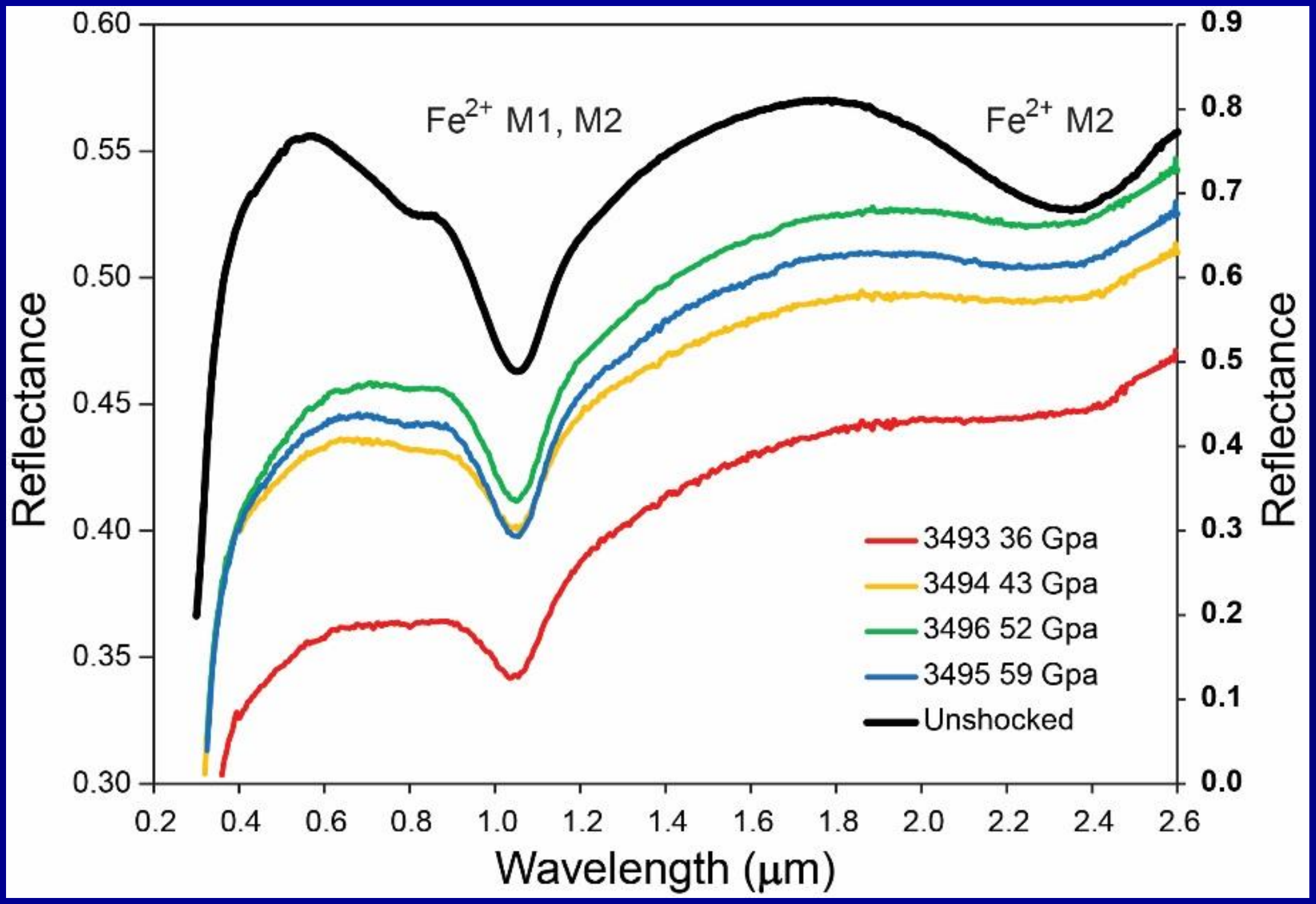

McCanta et al. (2016) Icarus, submitted 
Table 1. CRISM Spectral Parameter Summary Products ${ }^{\mathrm{a}}$

\begin{tabular}{|c|c|c|c|}
\hline Name & Parameter & Formulation $^{\mathrm{b}}$ & Rationale \\
\hline \multicolumn{4}{|c|}{ Surface Parameters ${ }^{\mathrm{c}}$} \\
\hline R770 & $0.77 \mu \mathrm{m}$ reflectance & $\mathrm{R} 770$ & rock/dust \\
\hline RBR & $\mathrm{red} / \mathrm{blue}$ ratio & R770/R440 & $\mathrm{rock} / \mathrm{dust}$ \\
\hline BD530 & $0.53 \mu \mathrm{m}$ band depth & $1-(\mathrm{R} 530 /(\mathrm{a} * \mathrm{R} 648+\mathrm{b} * \mathrm{R} 440))$ & crystalline ferric minerals \\
\hline SH600 & $0.60 \mu \mathrm{m}$ shoulder height & $\mathrm{R} 600 /(\mathrm{a} * \mathrm{R} 530+\mathrm{b} * \mathrm{R} 680)$ & select ferric minerals \\
\hline BD640 & $0.64 \mu \mathrm{m}$ band depth & $1-(\mathrm{R} 648 /(\mathrm{a} * \mathrm{R} 600+\mathrm{b} * \mathrm{R} 680))$ & select ferric minerals \\
\hline BD860 & $0.86 \mu \mathrm{m}$ band depth & $1-(\mathrm{R} 860 /(\mathrm{a} * \mathrm{R} 800+\mathrm{b} * \mathrm{R} 920))$ & select ferric minerals \\
\hline RPEAK1 & reflectance peak 1 & $\begin{array}{l}\text { wavelength where } 1 \text { st derivative }=0 \\
\text { of 5th order polynomial fit to R600, } \\
\text { R648, R680, R710, R740, R770, R800, R830 }\end{array}$ & Fe mineralogy \\
\hline BDI1000VIS & $\begin{array}{l}1 \mu \mathrm{m} \text { integrated band depth; } \\
\text { VIS wavelengths }\end{array}$ & $\begin{array}{l}\text { divide R830, R860, R890, R920 } \\
\text { by RPEAK } 1 \text { then integrate over } \\
(1-\text { normalized reflectances })\end{array}$ & Fe mineralogy \\
\hline BDI1000IR & $\begin{array}{l}1 \mu \mathrm{m} \text { integrated band depth; } \\
\text { IR wavelengths }\end{array}$ & $\begin{array}{l}\text { divide } \mathrm{R} 950, \mathrm{R} 980, \mathrm{R} 1020, \mathrm{R} 1050, \mathrm{R} 1080, \mathrm{R} 1150 \\
\text { by linear fit from peak R between } 1.3-1.87 \mu \mathrm{m} \\
\text { to } \mathrm{R} 2530 \text { extrapolated backward to remove } \\
\text { continuum, then integrate over } \\
\text { ( } 1-\text { continuum-corrected reflectances })\end{array}$ & Fe mineralogy \\
\hline IRA & $1.3 \mu \mathrm{m}$ reflectance & $\mathrm{R} 1330$ & IR albedo \\
\hline OLINDEX & Olivine index & $\begin{array}{l}(\mathrm{R} 1695 /(0.1 * \mathrm{R} 1050+0.1 * \mathrm{R} 1210 \\
\quad+0.4 * \mathrm{R} 1330+0.4 * \mathrm{R} 1470))-1\end{array}$ & $\begin{array}{l}\text { olivine will be strongly positive; } \\
\text { based on fayalite }\end{array}$ \\
\hline LCPINDEX & pyroxene index & $\begin{array}{l}((\mathrm{R} 1330-\mathrm{R} 1050) /(\mathrm{R} 1330+\mathrm{R} 1050)) \\
\quad *((\mathrm{R} 1330-\mathrm{R} 1815) /(\mathrm{R} 1330+\mathrm{R} 1815)\end{array}$ & $\begin{array}{l}\text { pyroxene will be strongly positive; } \\
\text { favors LCP }\end{array}$ \\
\hline HCPXINDEX & pyroxene index & $\begin{array}{l}((\mathrm{R} 1470-\mathrm{R} 1050) /(\mathrm{R} 1470+\mathrm{R} 1050)) \\
\quad *((\mathrm{R} 1470-\mathrm{R} 2067) /(\mathrm{R} 1470+\mathrm{R} 2067)\end{array}$ & $\begin{array}{l}\text { pyroxene will be strongly positive; } \\
\text { favors HCP }\end{array}$ \\
\hline VAR & spectral variance & variance of observed data from a line fit from $1.0-2.3 \mu \mathrm{m}$ & $\begin{array}{l}\text { olivine and pyroxene will have } \\
\text { high values }\end{array}$ \\
\hline ISLOPE1 & $-1 *$ spectral slope 1 & $(\mathrm{R} 1815-\mathrm{R} 2530) /(2530-1815)$ & ferric coating on dark rock \\
\hline BD1435 & $1.435 \mu \mathrm{m}$ band depth & $1-(\mathrm{R} 1430 /(\mathrm{a} * \mathrm{R} 1370+\mathrm{b} * \mathrm{R} 1470))$ & $\mathrm{CO}_{2}$ ice \\
\hline
\end{tabular}
diversity on Mars from reflectance, J. Geophys. Res., 112, E08S14, doi:10.1029/2006JE002831. 


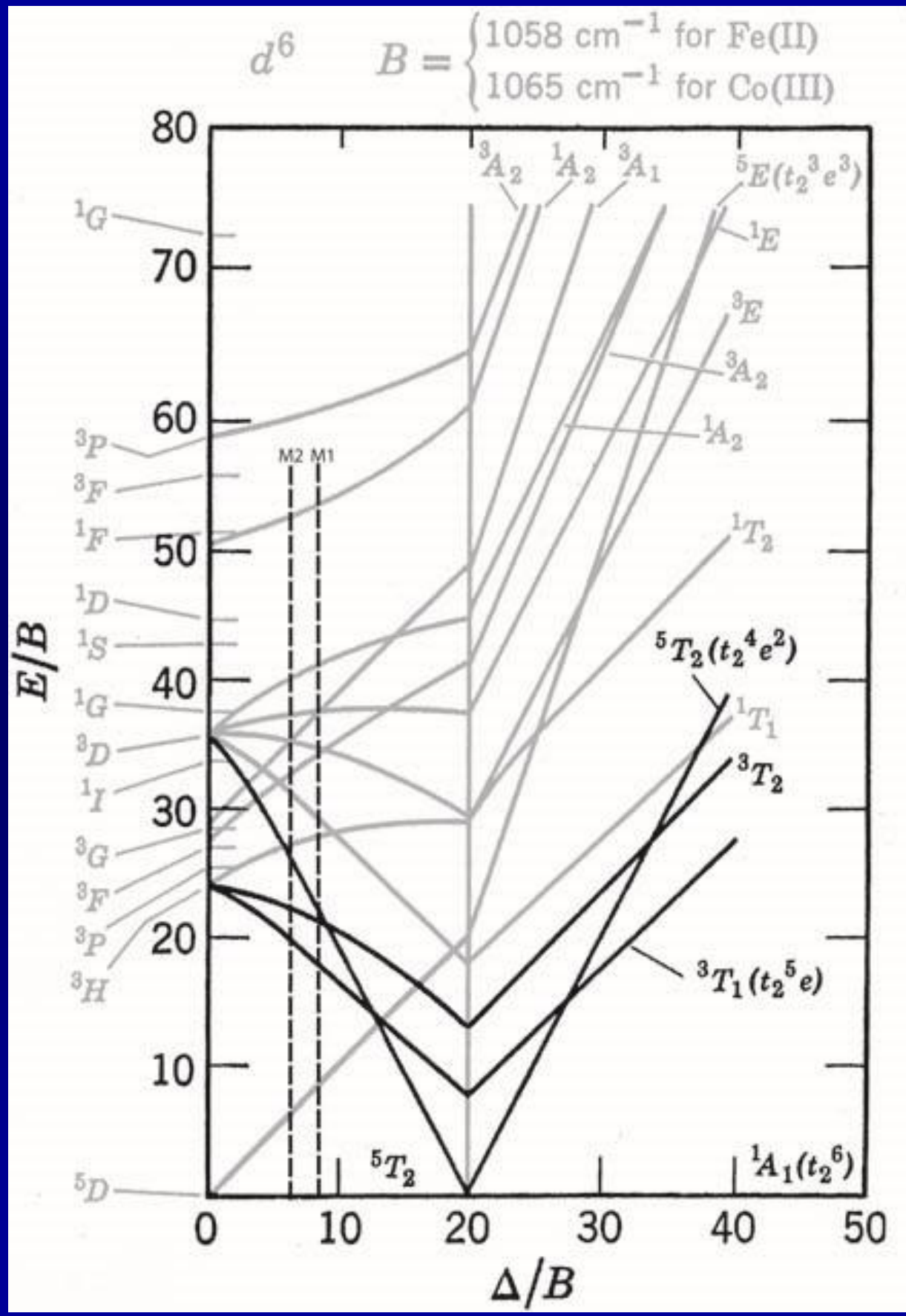

\section{Tanabe- Sugano diagram for pyroxene} MAPS, 42, 235-253 
Spectroscopy $+$

Machine

Learning

$$
=
$$

Better

Spectroscopy

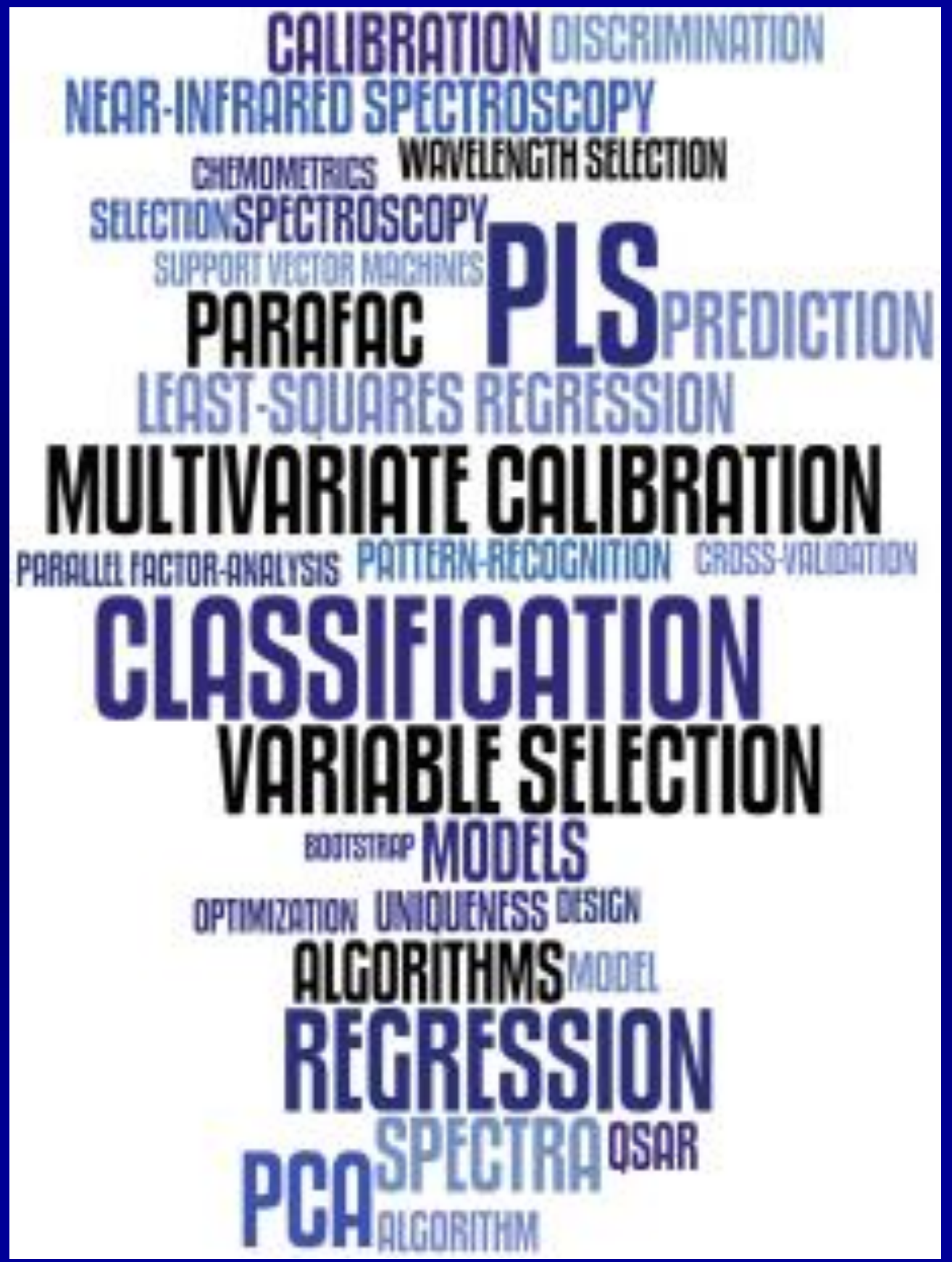

http://onlinelibrary.wiley.com/journal/10.1002/\%28ISSN\%291099-128X 
Chemometrics is an interdisciplinary field combining experimental design, physical-chemical measurements, multivariate statistical analysis, mathematical modeling, and information technology for extracting useful information from data.

...Journal of Chemometrics

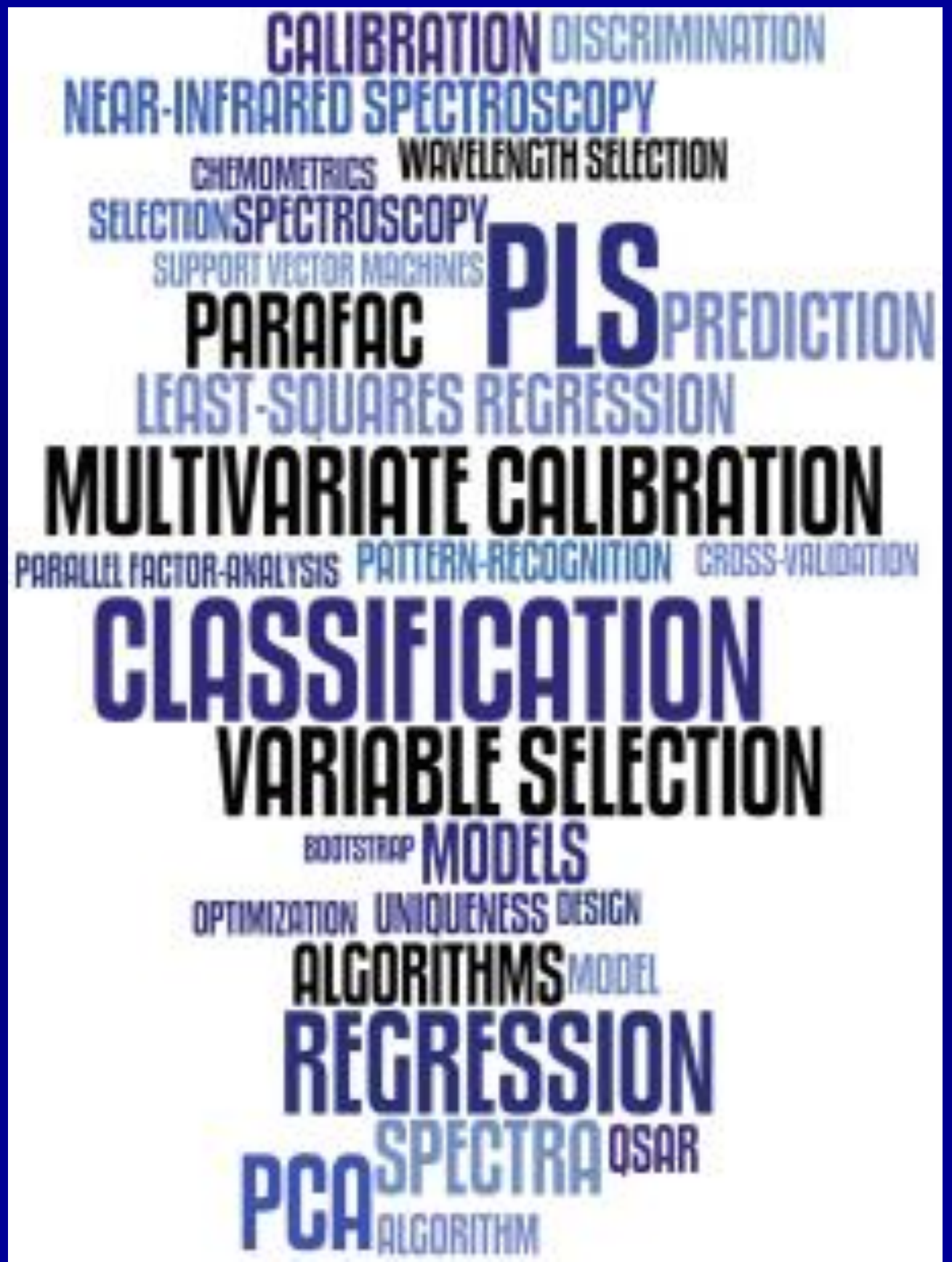




\section{Chemometric Approaches to:}

A. Multivariate analysis

B. X-ray absorption spectroscopy

C. Laser-induced breakdown spectroscopy

D. Baseline removal

E. Calibration transfer

F. Data preprocessing 


\section{Most Basic Technique for Multivariate Analysis Partial Least Squares (PLS)}

- Shrink regression equation by creating hybrid channels that are linear combinations of all previous channels.

- Correlate two matrices described by $\mathbf{Y}=\mathbf{X} \mathbf{b}$ :

- Spectra $(X)$ ( $\boldsymbol{p}$ samples $\times \mathbf{N}$ channels)

- Variable(s) of interest (Y)

- This removes co-linearity because directions in that new vector space are ortho-normal, avoiding the problem that inhibits ordinary least-squares regression.

- PLS analysis thus produces $b$-coefficients for each channel that represent the correlation implicit in $\mathbf{b}$. 


\section{Most Basic Technique for Multivariate Analysis Partial Least Squares (PLS)}

Each Spectral Channel is an Independent Variable Prediction Quantities (elements, mineralogy, $\% \mathrm{Fe}^{3+)}$ are the Dependent Variable(s)

\begin{tabular}{|l|c|c|c|c|c|c|c|c|c|c|c|c|c|} 
& $\mathrm{Fe}^{3+}$ & $\begin{array}{c}\lambda 1 \\
(\mathrm{keV})\end{array}$ & $\begin{array}{c}\lambda 2 \\
(\mathrm{keV})\end{array}$ & $\begin{array}{c}\lambda 3 \\
(\mathrm{keV})\end{array}$ & $\begin{array}{c}\lambda 4 \\
(\mathrm{keV})\end{array}$ & $\begin{array}{c}\lambda 5 \\
(\mathrm{keV})\end{array}$ & $\begin{array}{c}\lambda 6 \\
(\mathrm{keV})\end{array}$ & $\begin{array}{c}\lambda 7 \\
(\mathrm{keV})\end{array}$ & $\begin{array}{c}\lambda 8 \\
(\mathrm{keV})\end{array}$ & $\begin{array}{c}\lambda 9 \\
(\mathrm{keV})\end{array}$ & $\begin{array}{c}\lambda 10 \\
(\mathrm{keV})\end{array}$ & $\ldots \ldots .$. & $\begin{array}{c}\lambda \mathrm{n} \\
(\mathrm{keV})\end{array}$ \\
\hline Sample 1 & & & & & & & & & & & & & \\
\hline Sample 2 & & & & & & & & & & & & & \\
\hline Sample 3 & & & & & & & & & & & & & \\
\hline Sample 4 & & & & & & & & & & & & \\
\hline Sample 5 & & & & & & & & & & & & & \\
\hline$\ldots$ & & & & & & & & & & & & & \\
\hline Sample $\mathrm{n}$ & & & & & & & & & & & & & \\
\hline
\end{tabular}




\section{Example \#1: \\ X-ray Absorption Spectroscopy}

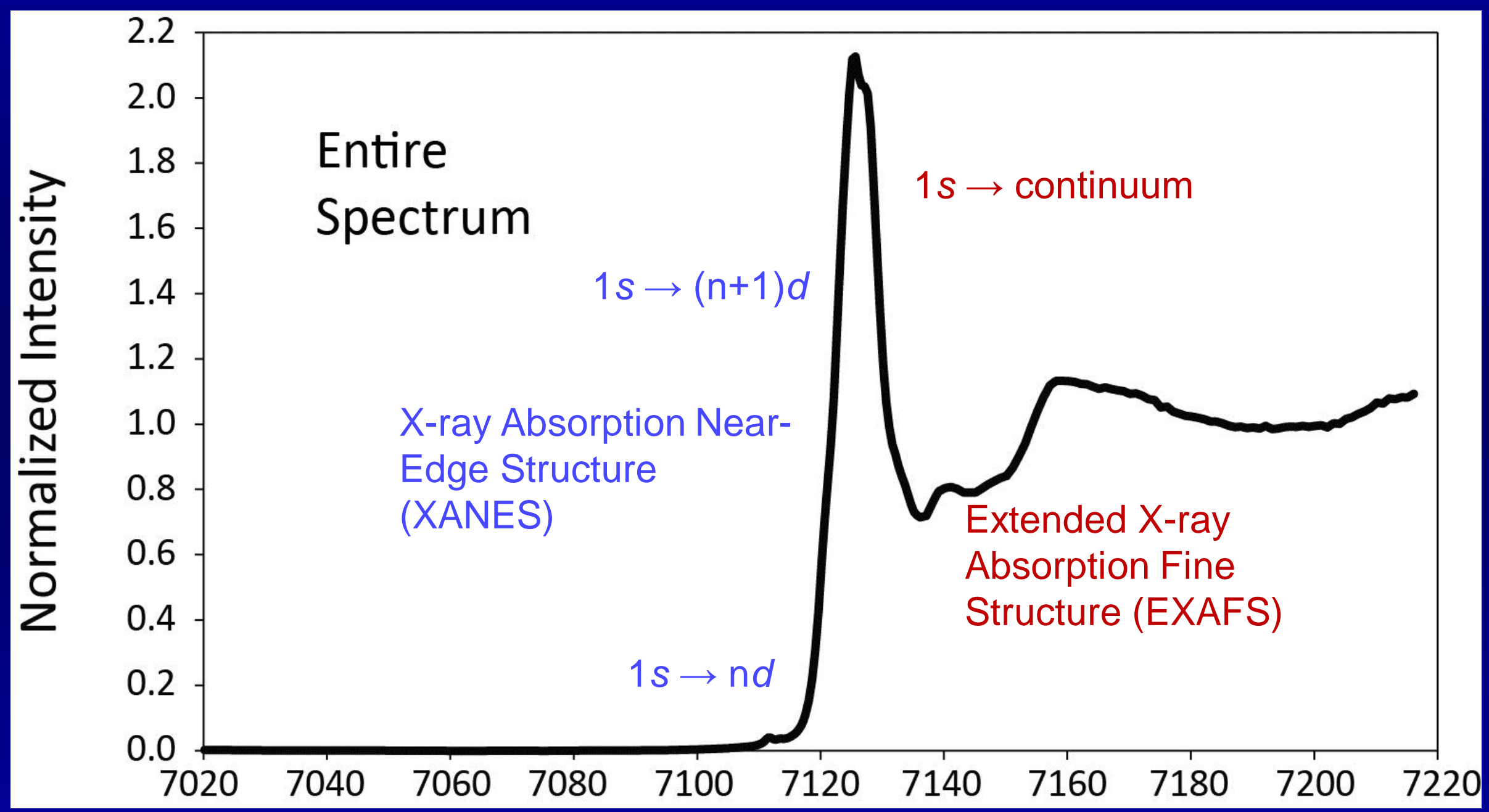




\section{Using XAS to measure redox state of $\mathrm{Fe}$}
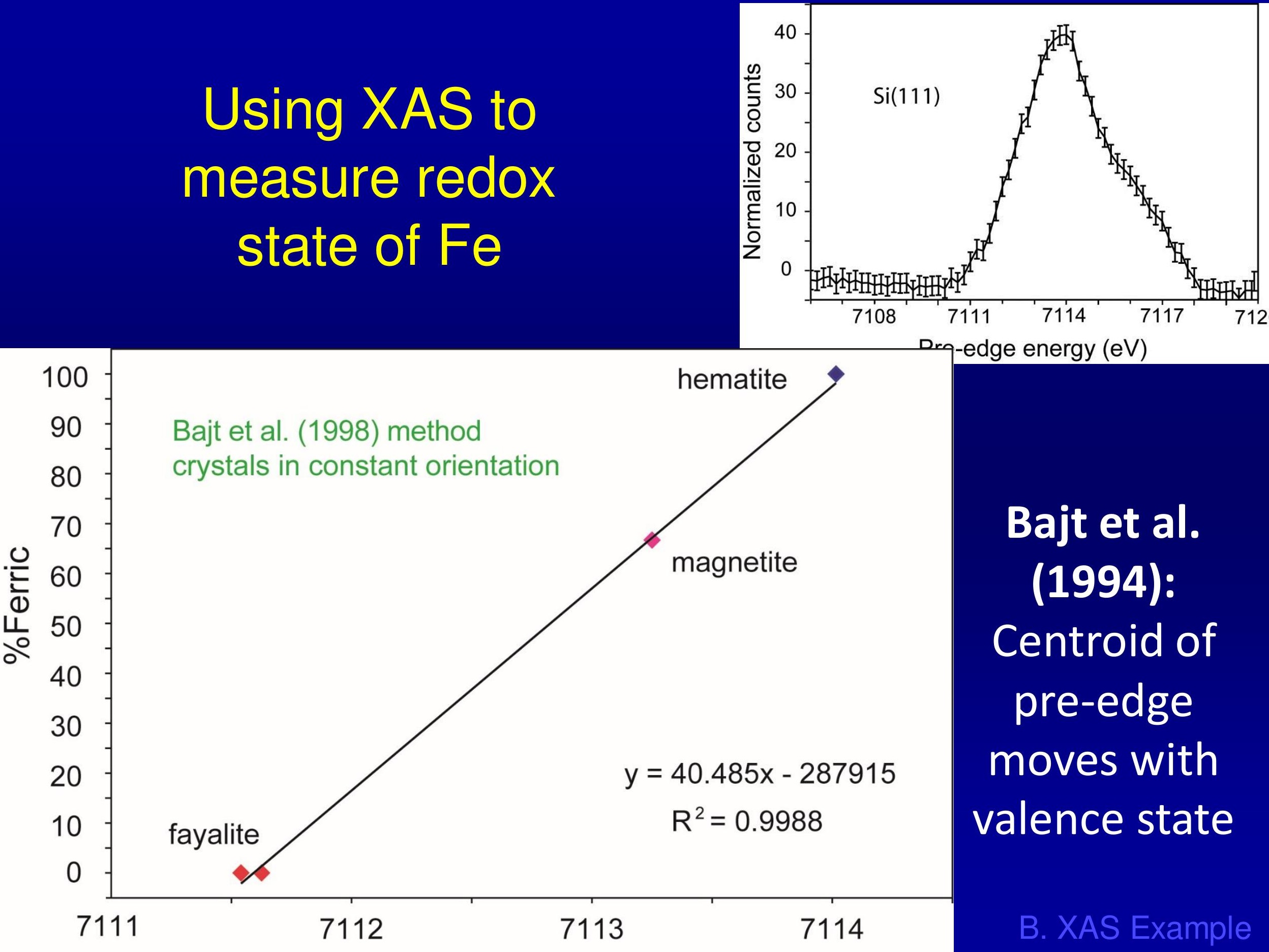
Fe XANES Data used to Predict $\% \mathrm{Fe}^{3+}$ in Powders using XANES Pre-Edge
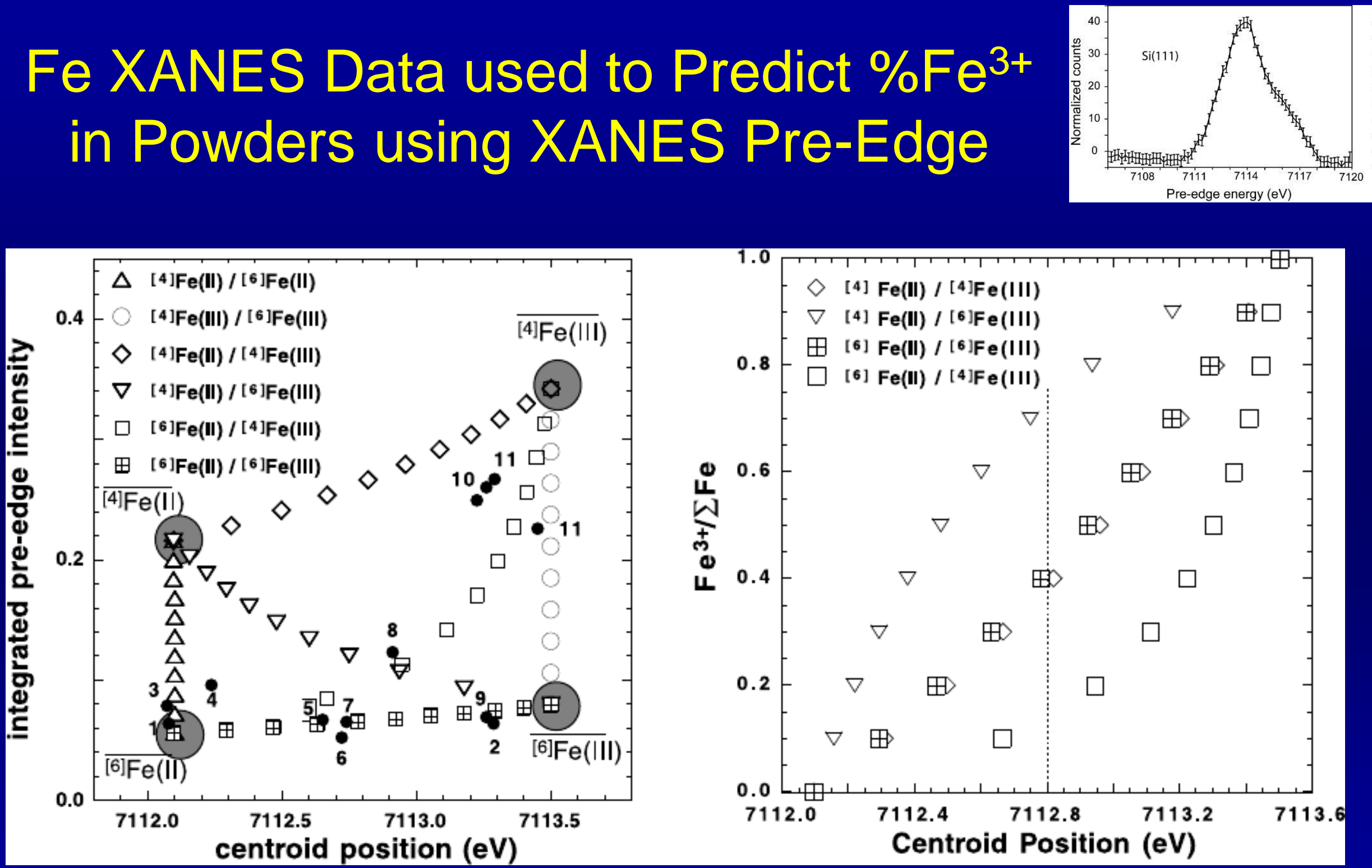

Wilke et al. (2001) Am. Min. 86, 714-730, calibration for powders 


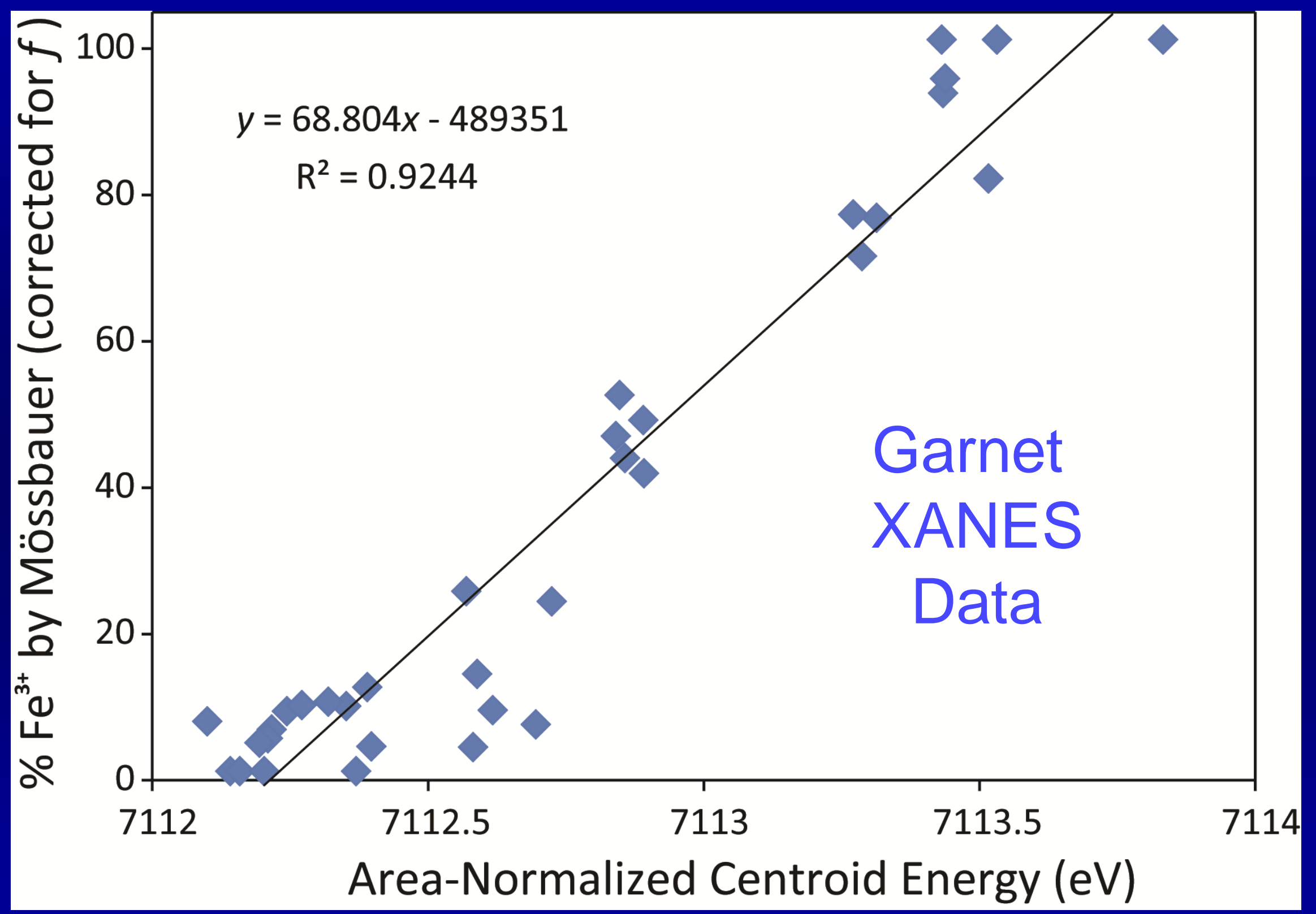




\section{Spectra of Oriented Crystals}

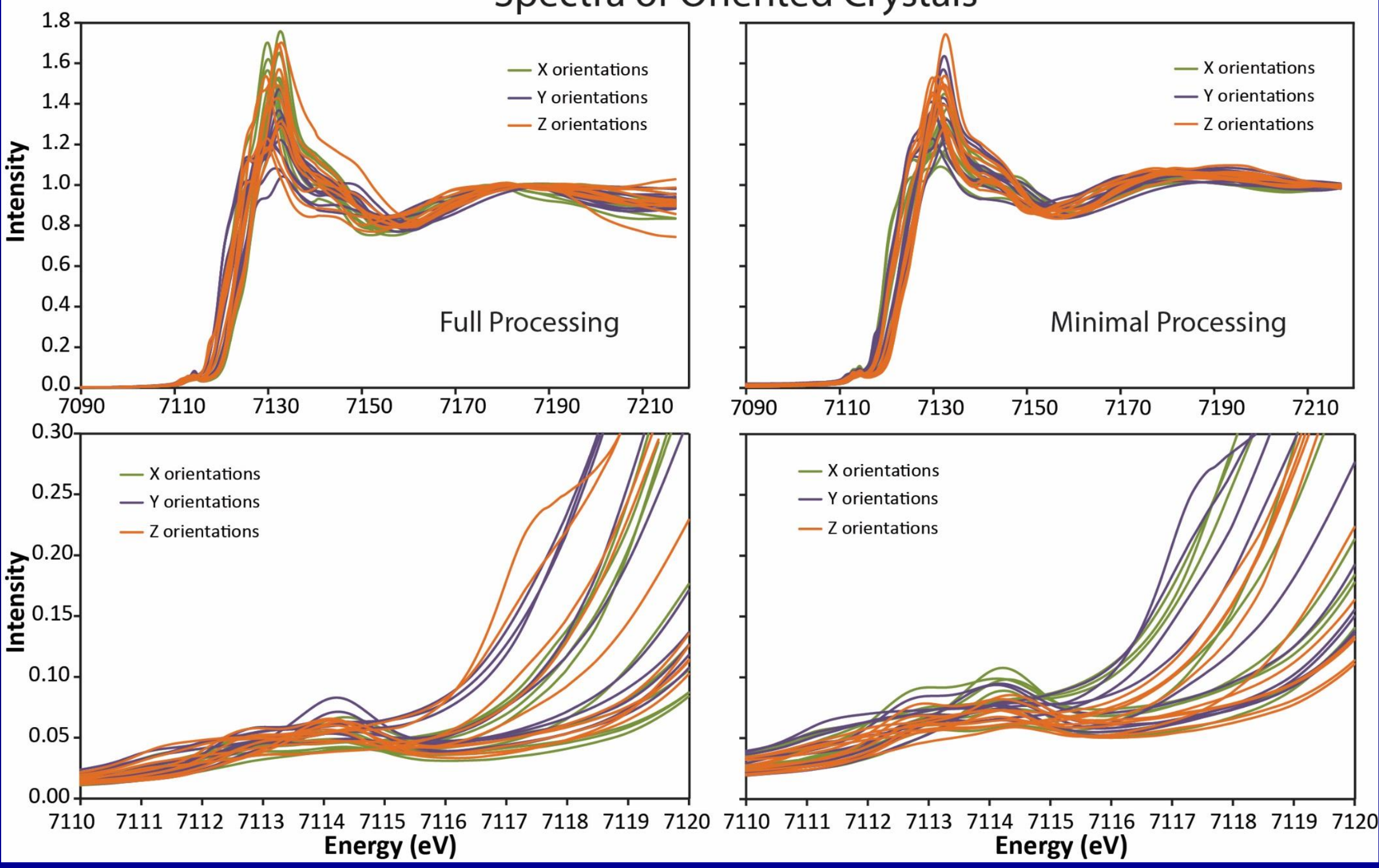

B. XAS Example 


\section{Multivariate \\ Predictions of $\mathrm{Fe}^{3+}$ in \\ Silicate Glass and \\ Garnet}
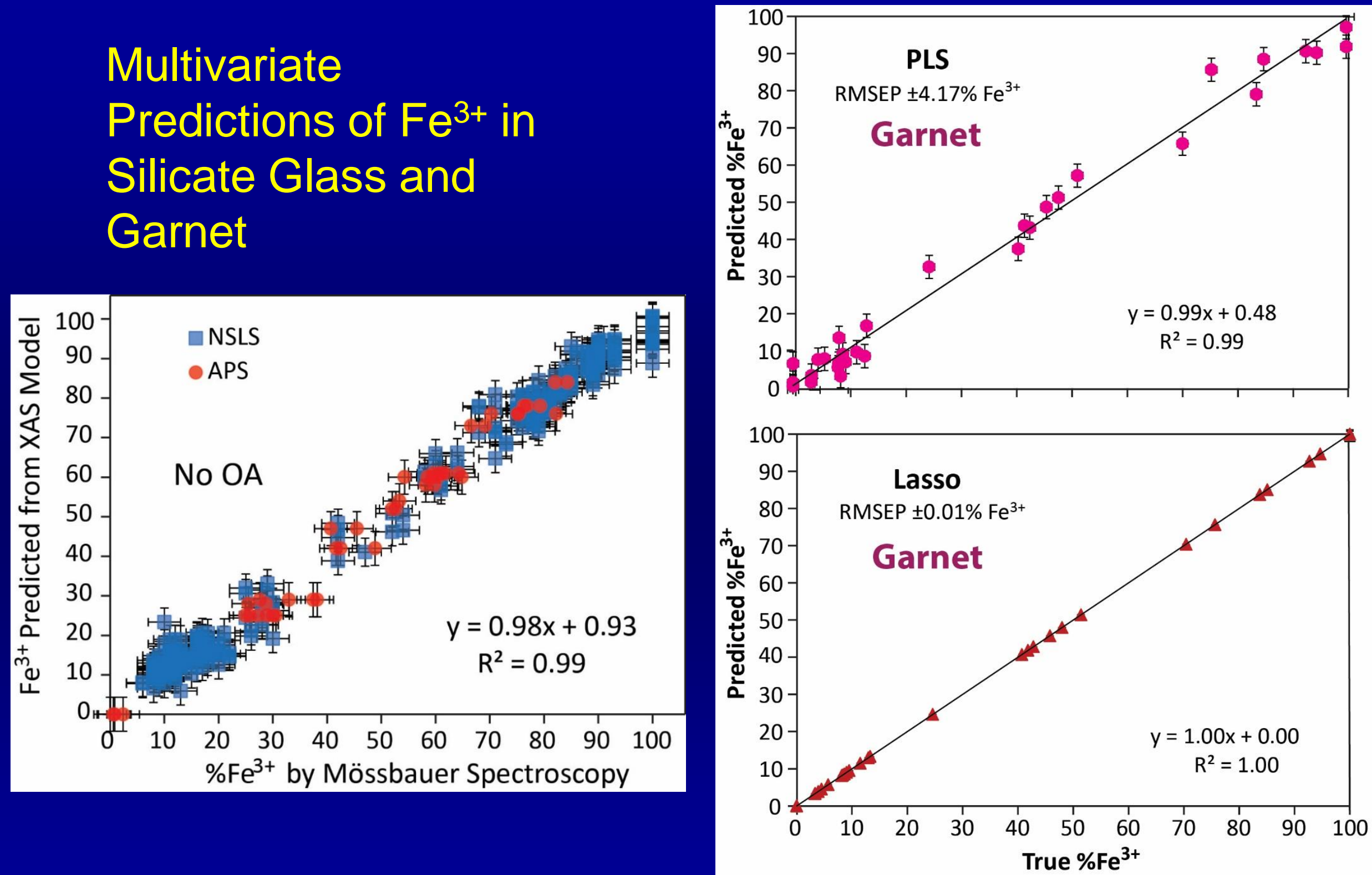


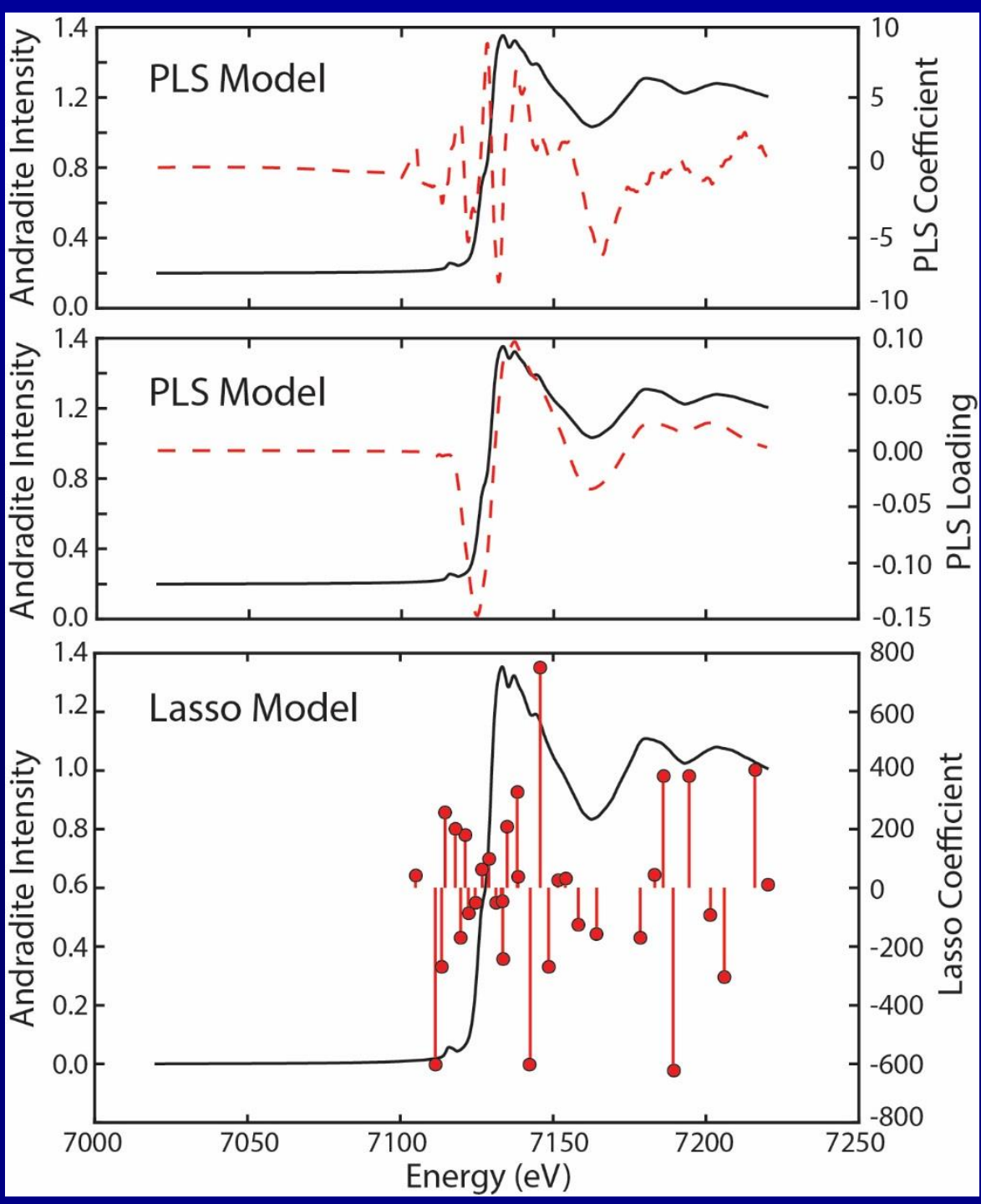

\section{Identification of Key Predictive Channels}

\section{Amphibole XAS Data}

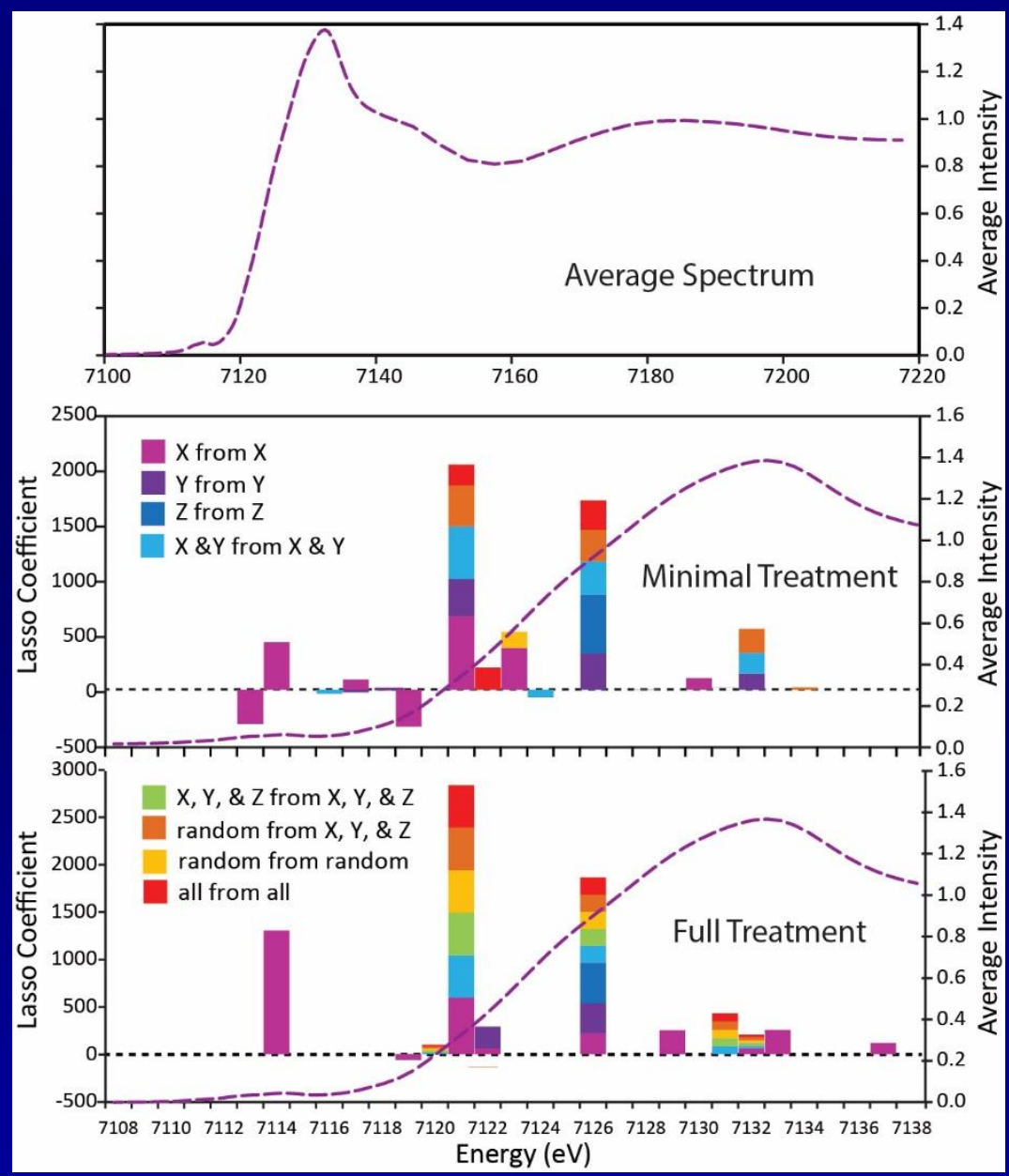

Garnet XAS Data 


\section{Laser-Induced Breakdown}

\section{Spectroscopy: LIBS}

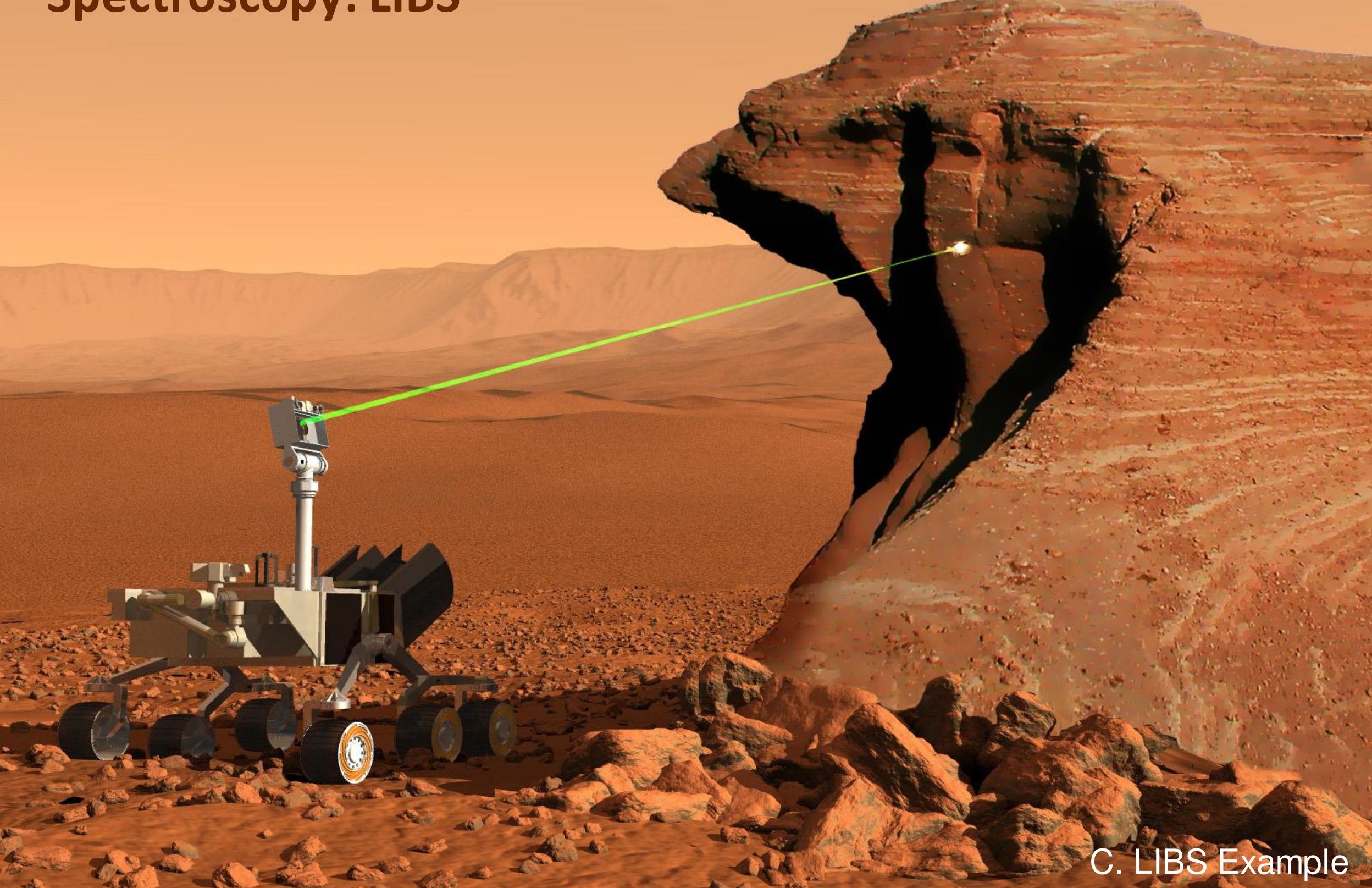




\section{Calibration curve and the best-fit line}

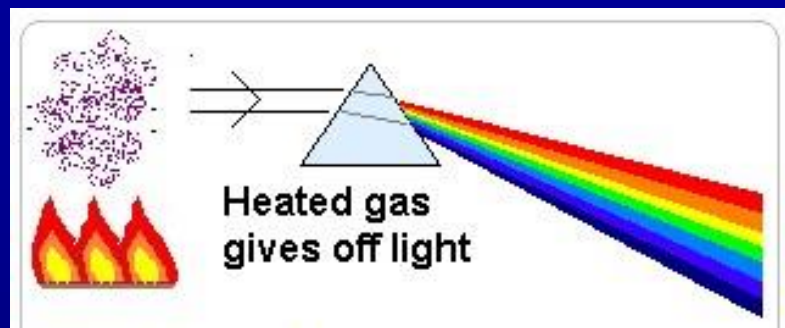

Which gives this:
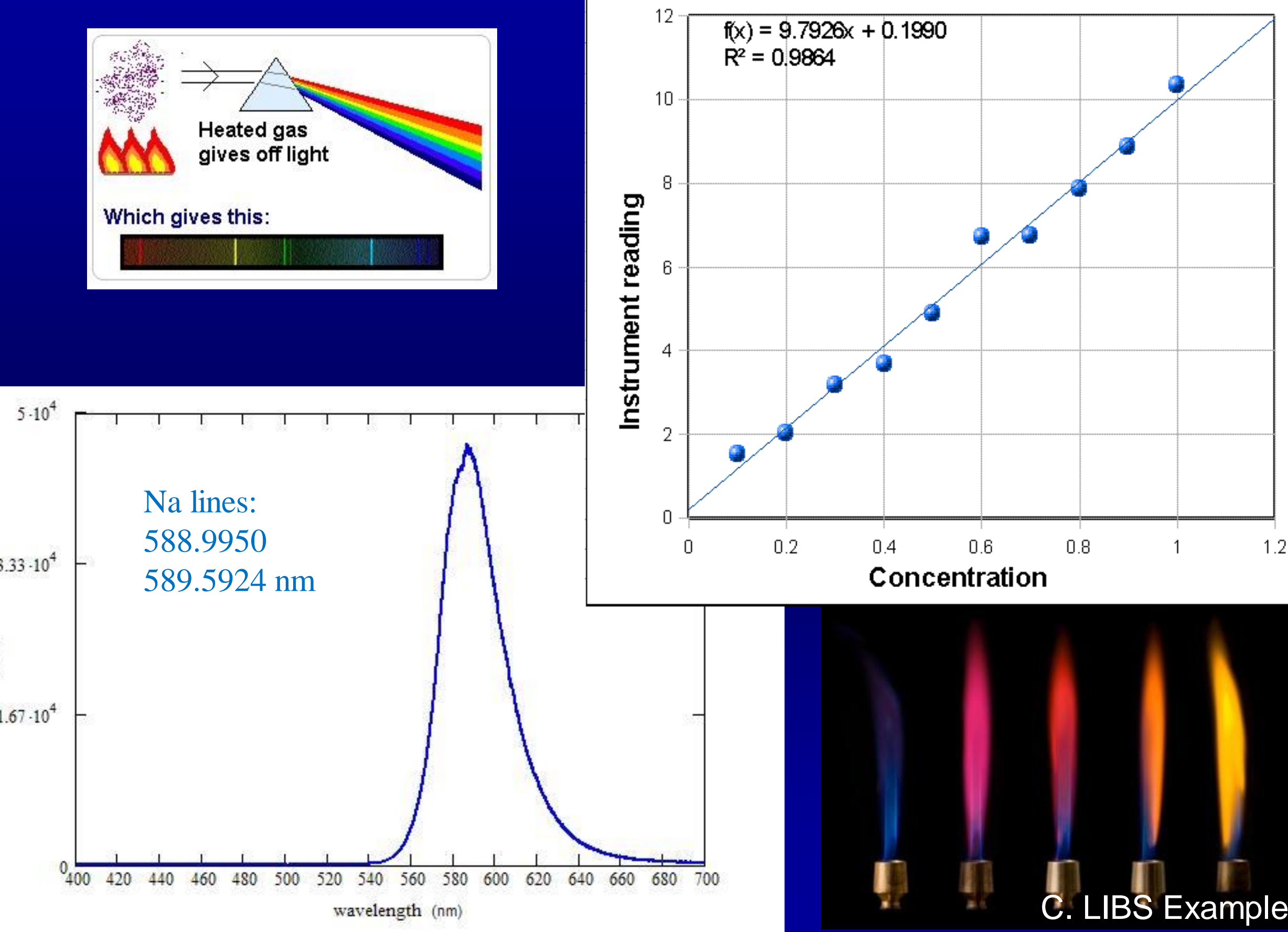

\section{Concentration}

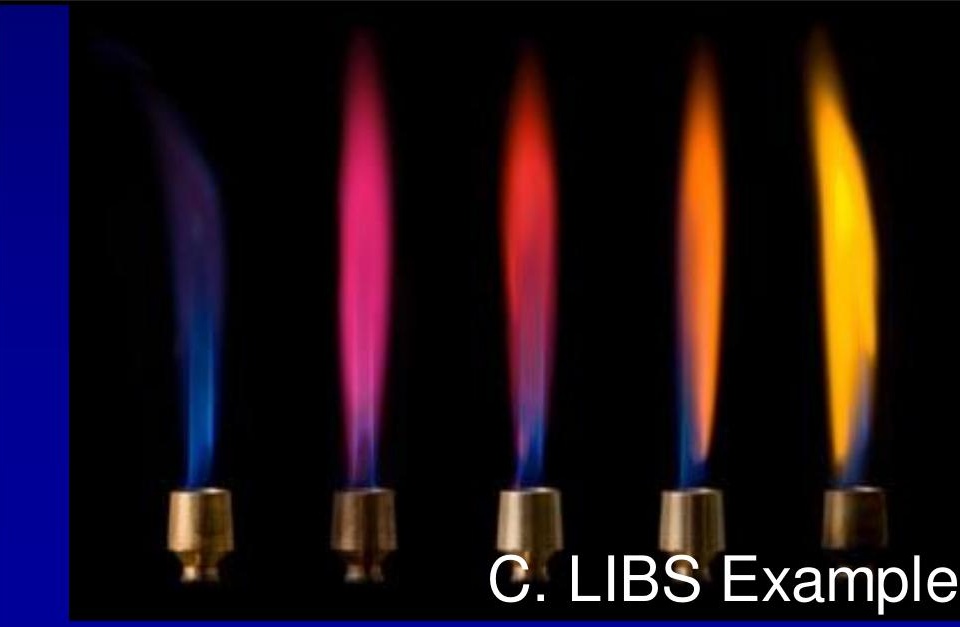




\section{LIBS Challenge for Geological Samples: Matrix Effects!}

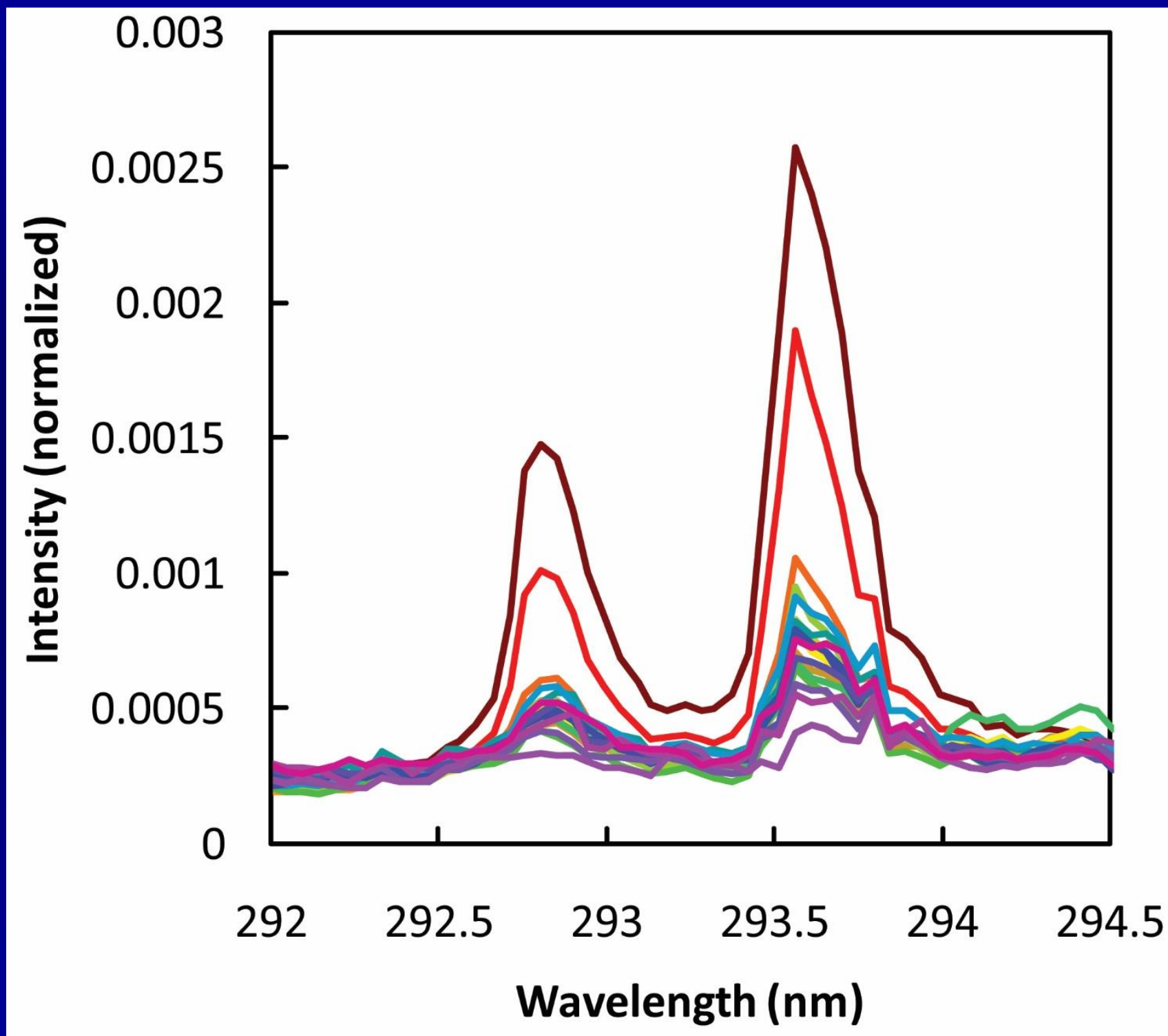

Ultrama

$\longrightarrow$ VH-49

Cerro

Moppin

$\longrightarrow 1984$ AA

GranoDike

Atascosa

BWQF-1

BWQC-1

WMG

$\longrightarrow$ MSHA

BK-2

$\longrightarrow$ Red Hill

$\longrightarrow$ Pipe Creek

$\longrightarrow \mathrm{VH}-1$

Umphr

$\longrightarrow$ Cadillac

Baveno

$\longrightarrow$ Trondj
High Ni

Low Ni 


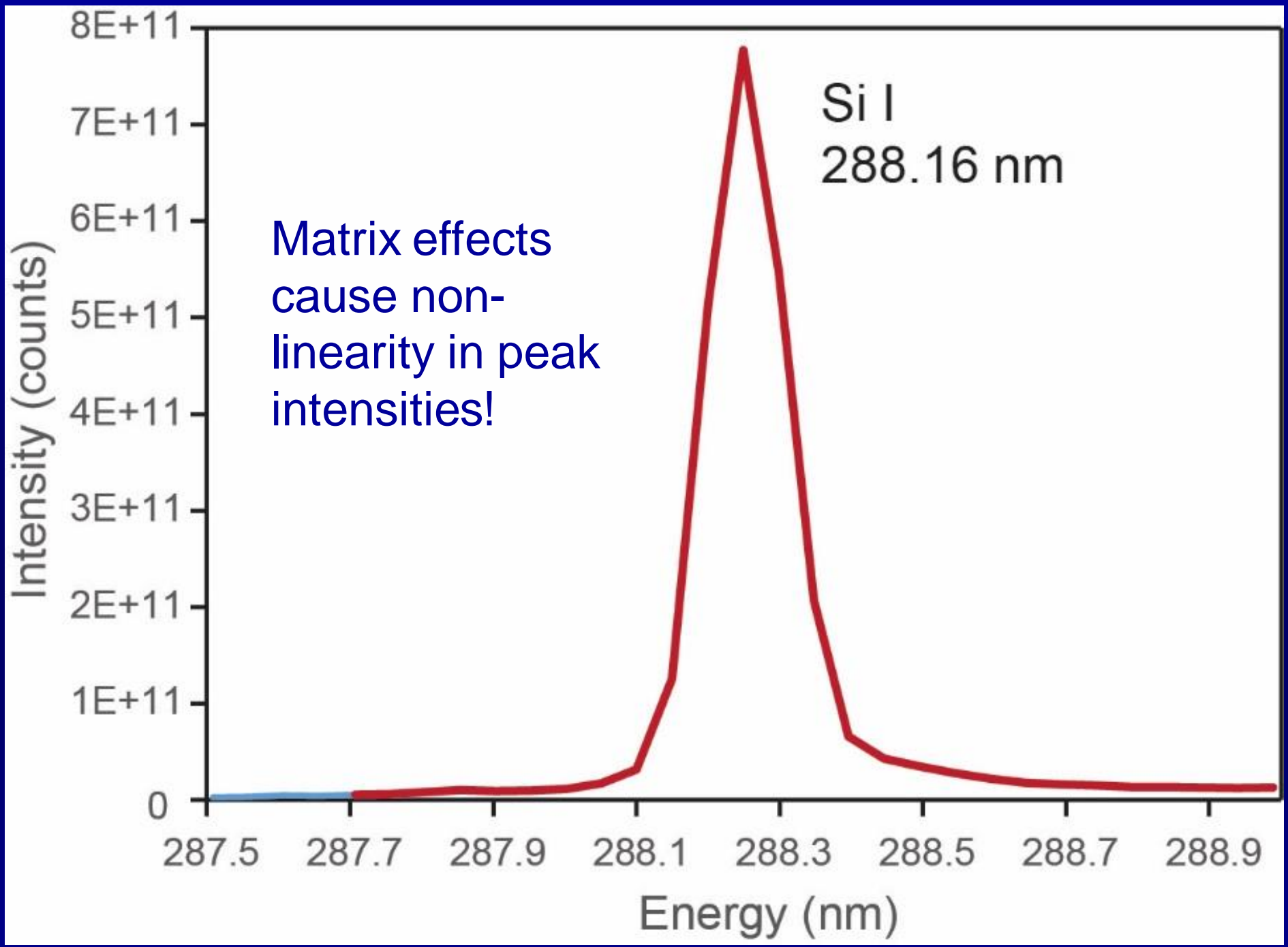

Prediction of $\mathrm{SiO}_{2}$ contents of LIBS standards (1354 samples, 3 plasma temperatures, Mars conditions) using this single Si I emission line is:

$$
\pm 13.76 \text { wt. } \% \mathrm{SiO}_{2}
$$




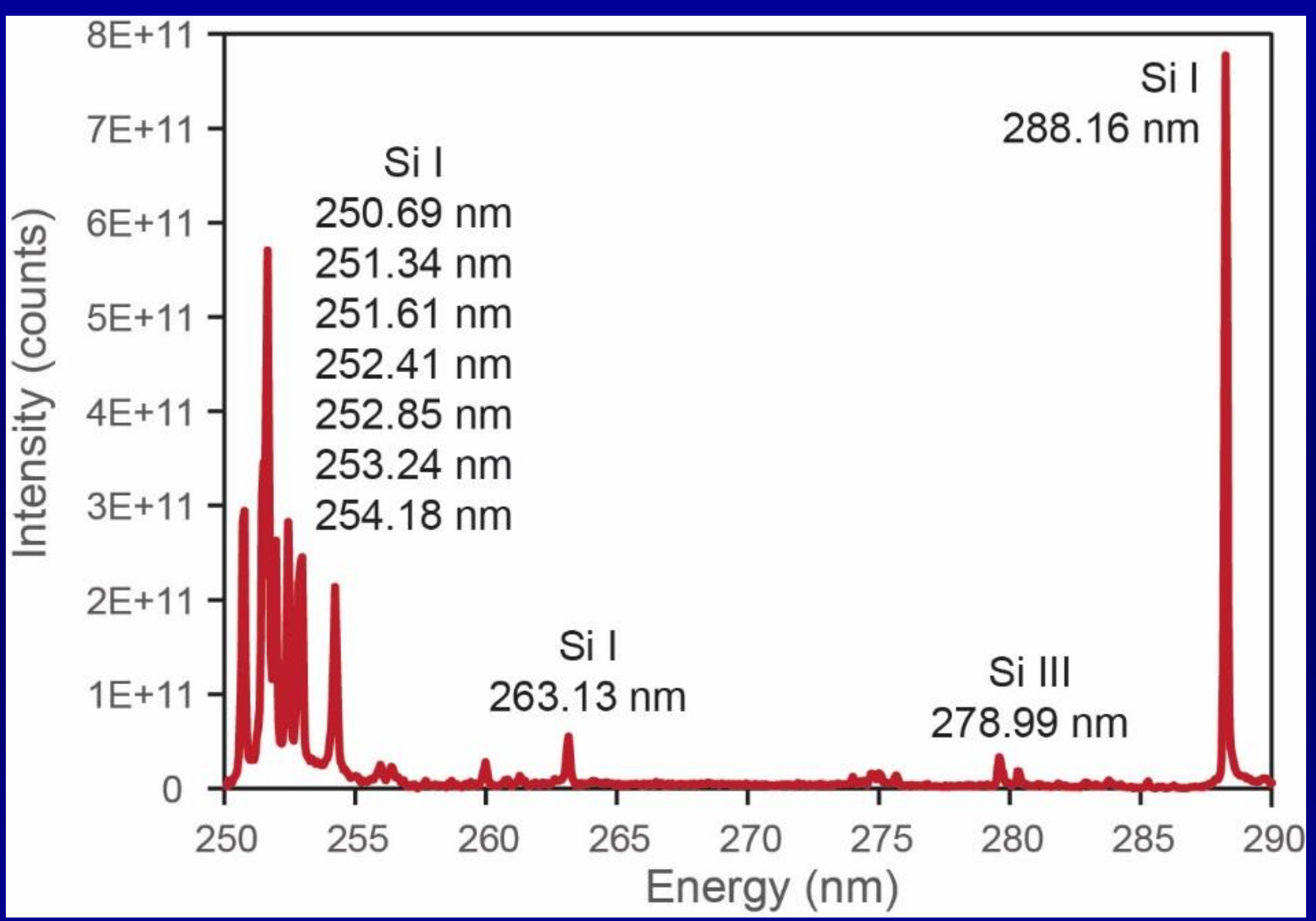

Prediction of $\mathrm{SiO}_{2}$ using all channels of this spectrum is: \pm 4.69 wt. $\% \mathrm{SiO}_{2}$ 
LIBS Prediction Accuracy Using One Peak vs. Entire Spectrum

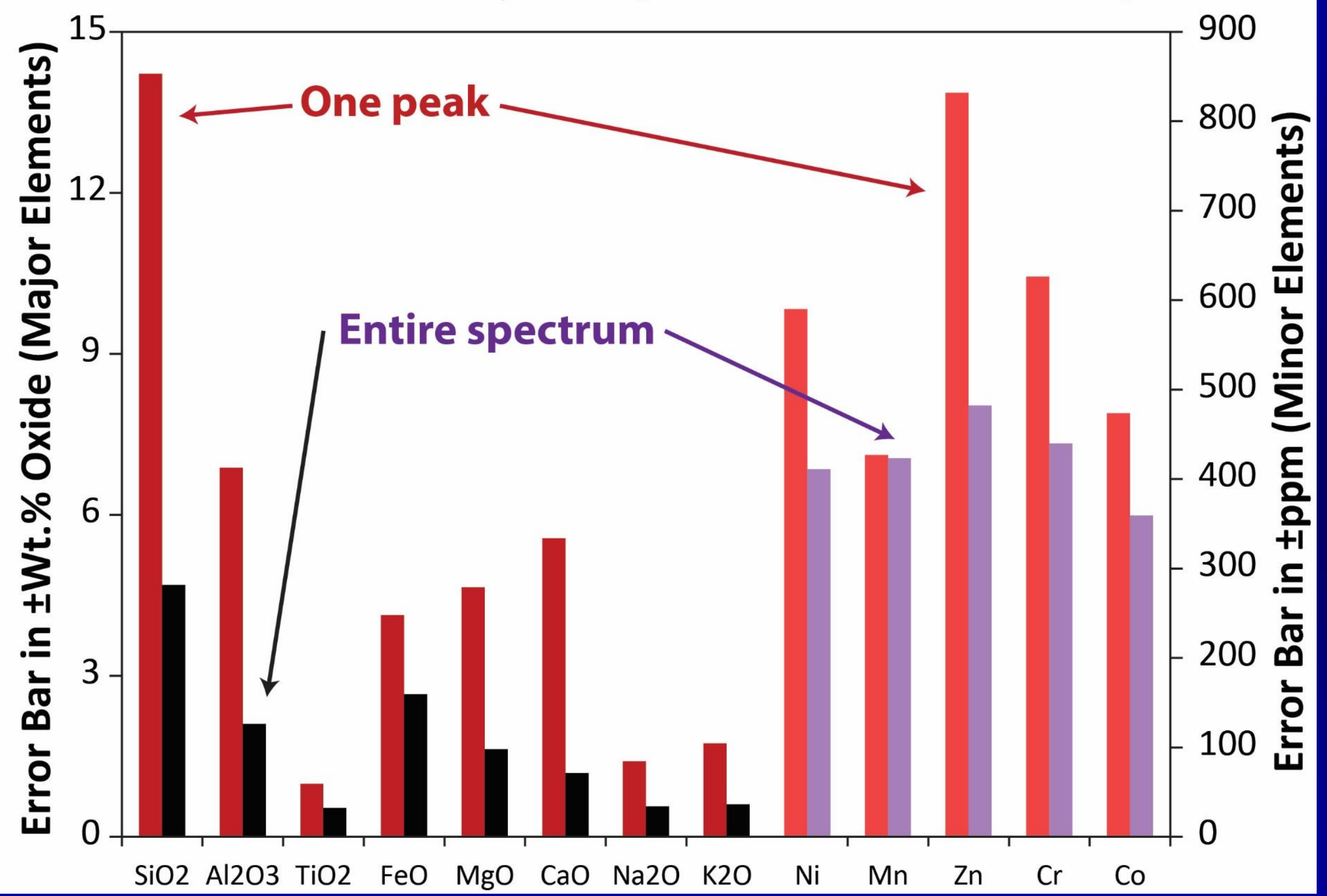

C. LIBS Example 


\section{Chemometrics (Machine Learning) gives us answers to vexing problems...}
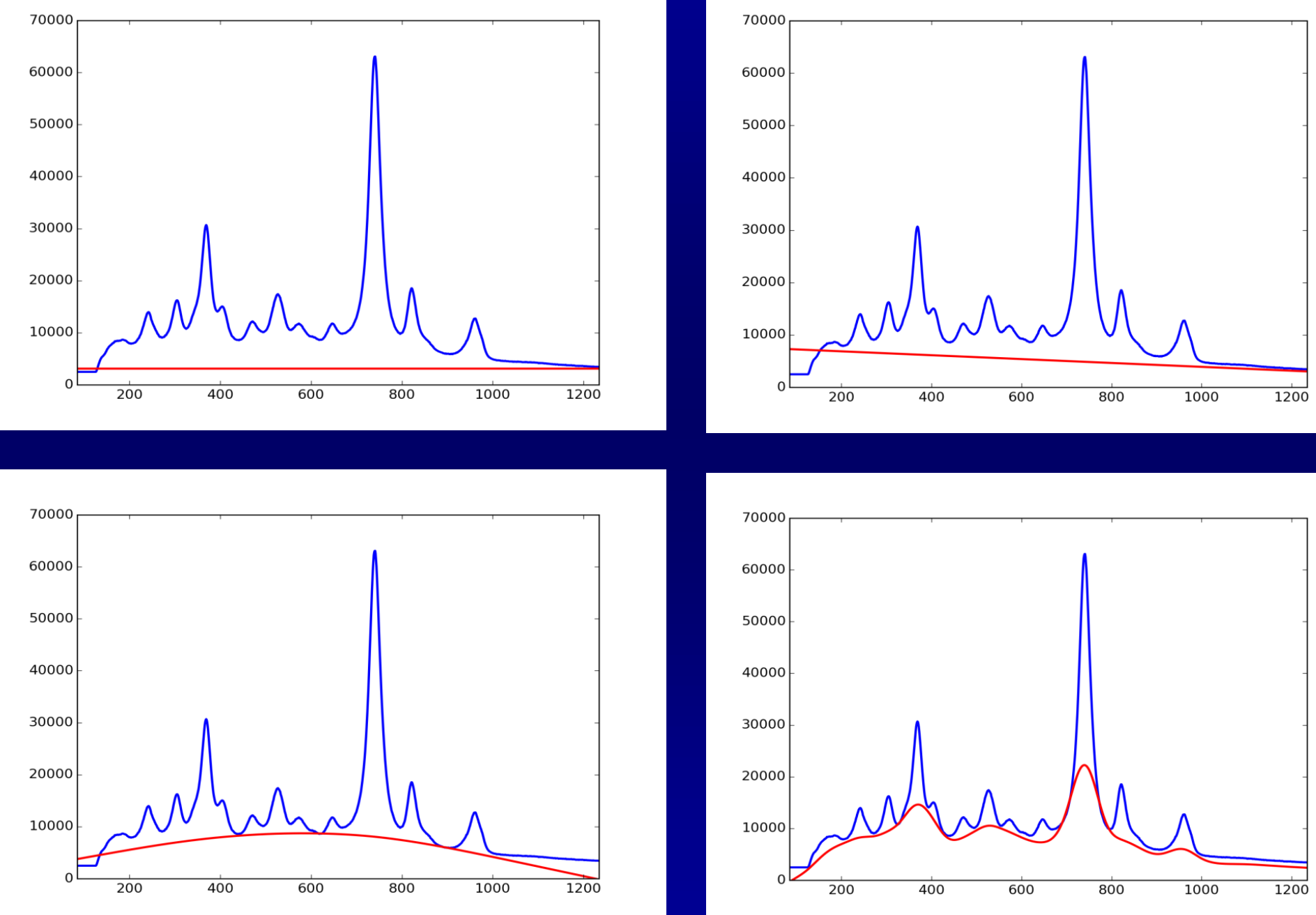

The baseline removal conundrum 


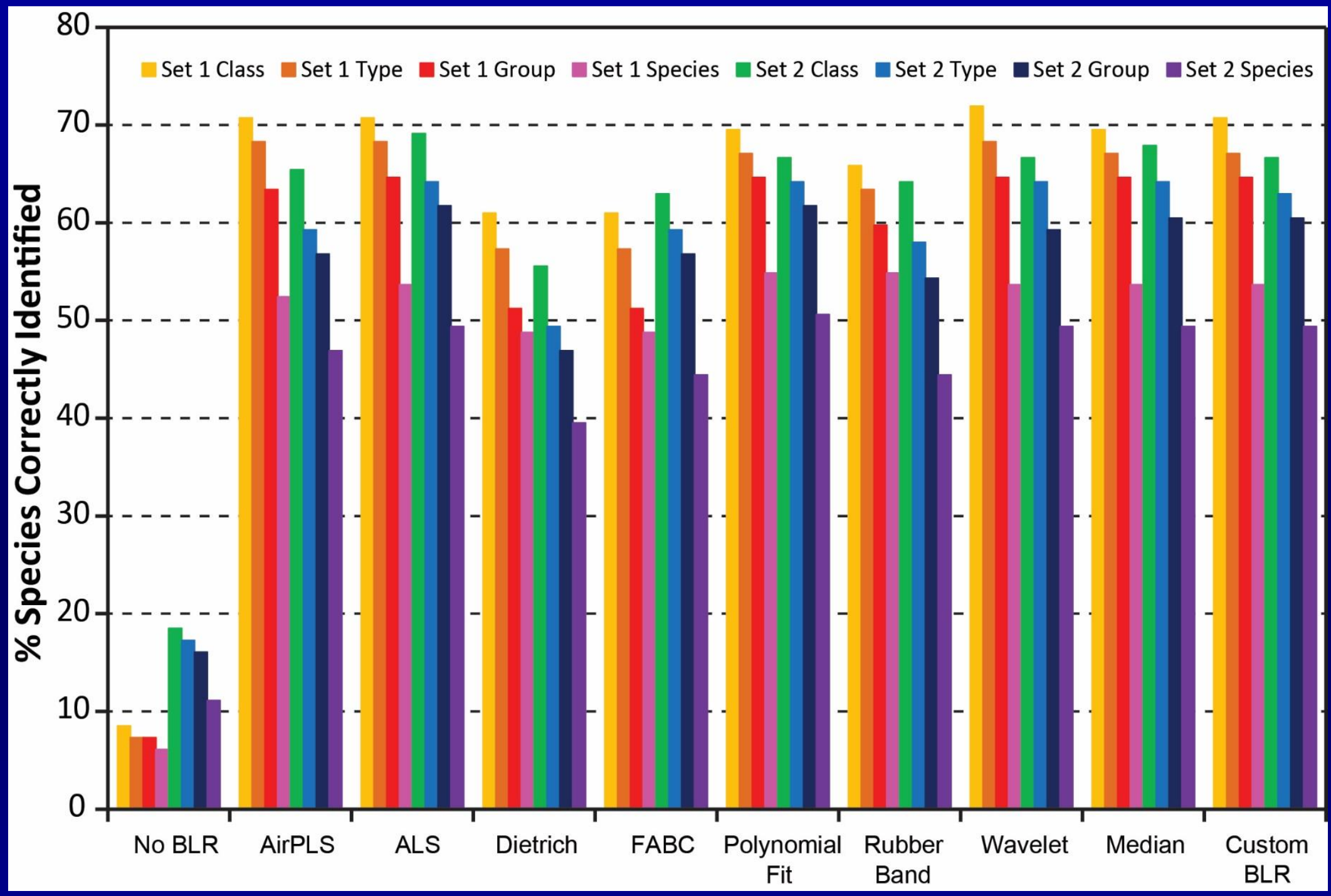

Species matching success for Raman spectroscopy comparing optimized baseline removal methods to no baseline removal (far left) and Custom BLR (far right) by taxonomic rank (Dana classification number). 

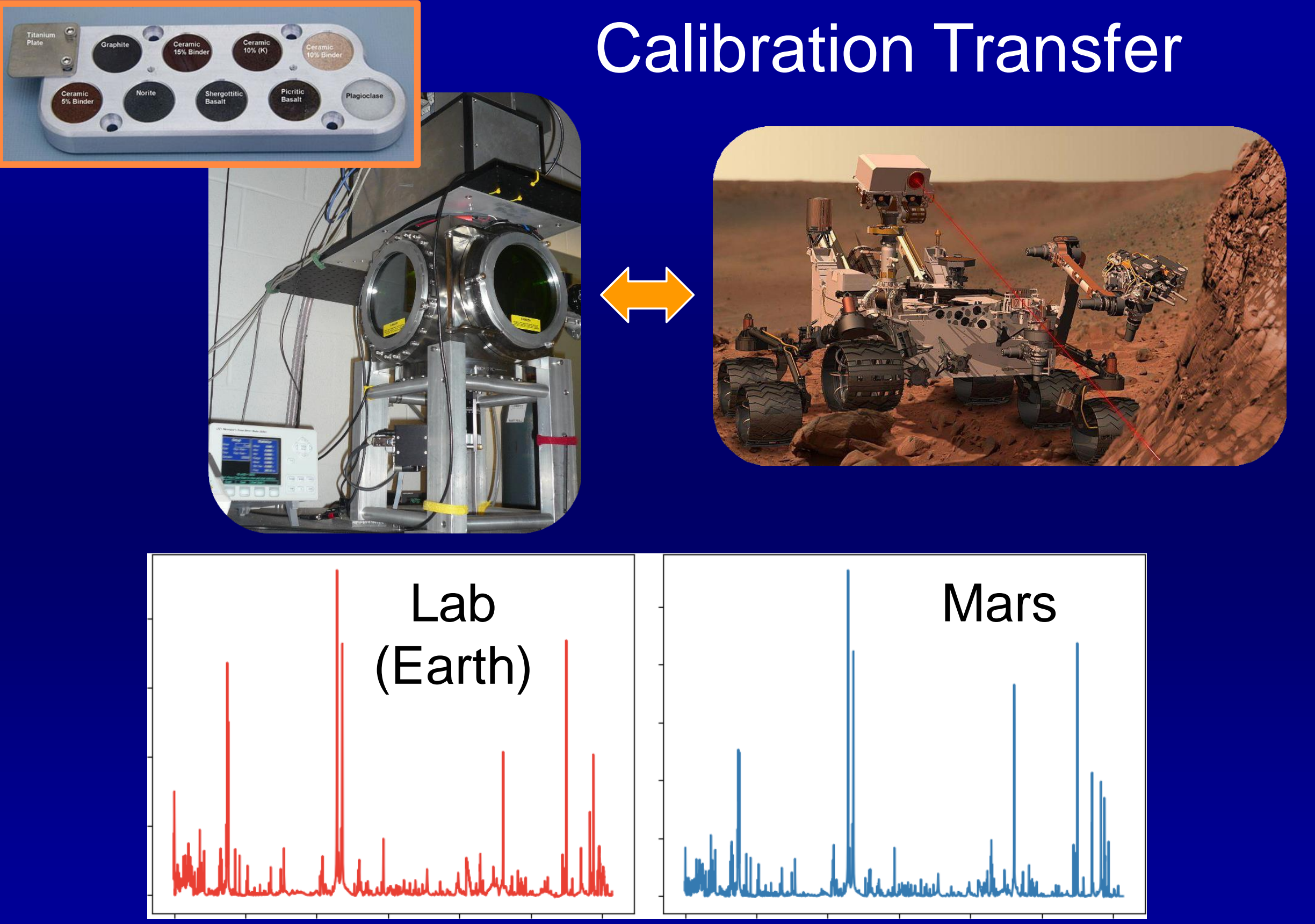

E. Cal Trans 


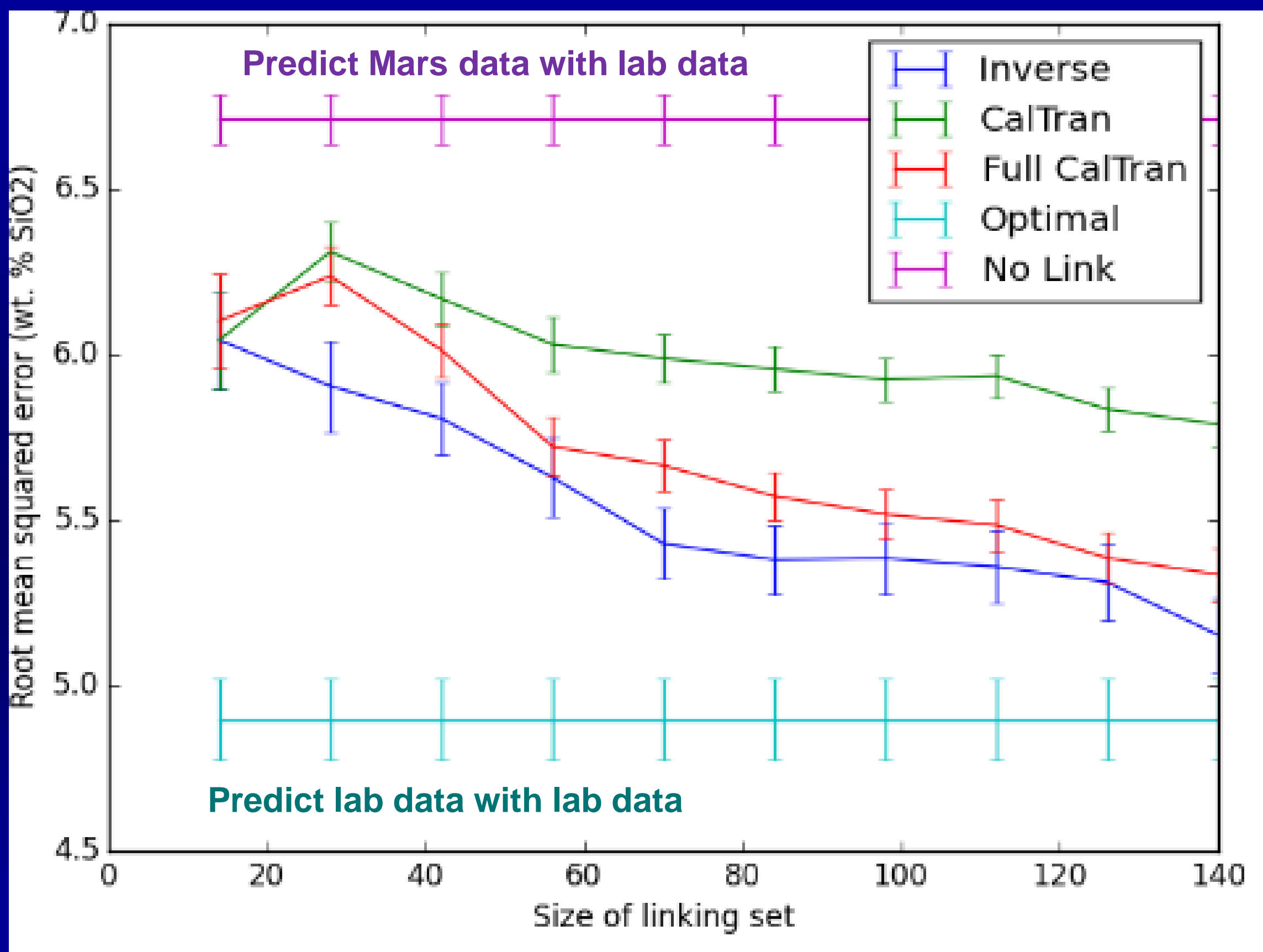

E. Cal Trans 


\section{Visualization of Spectral Preprocessing Steps}

\section{Original}

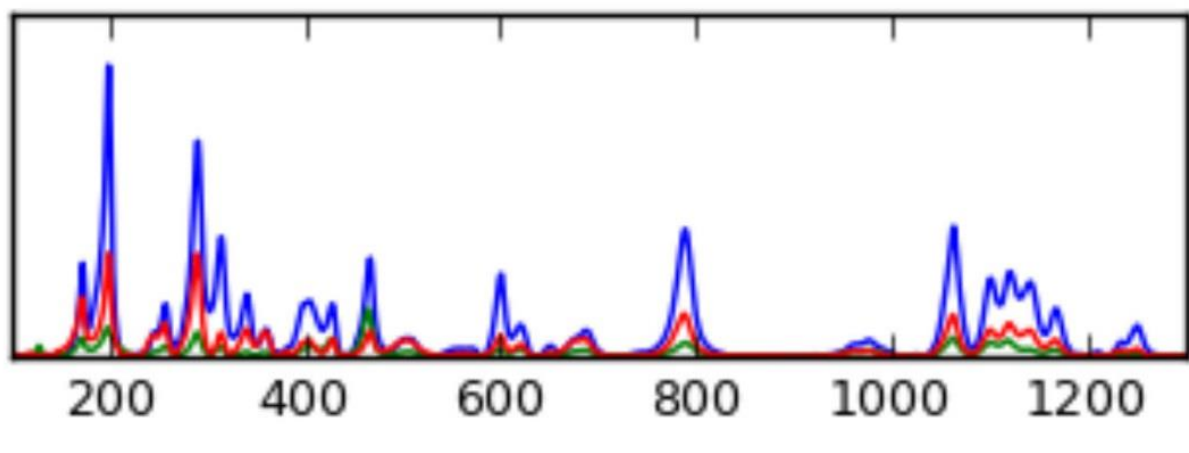

Squashed

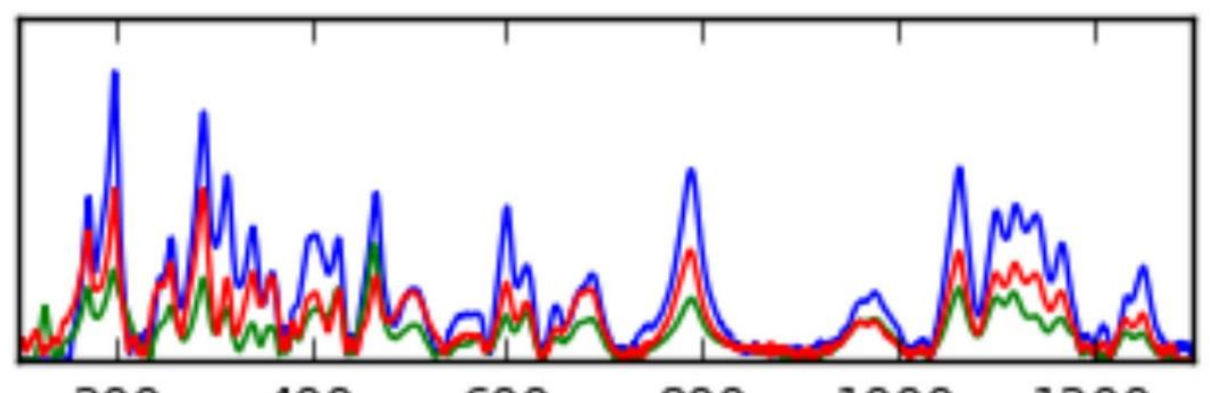

200 400 600 10001200
Normalized

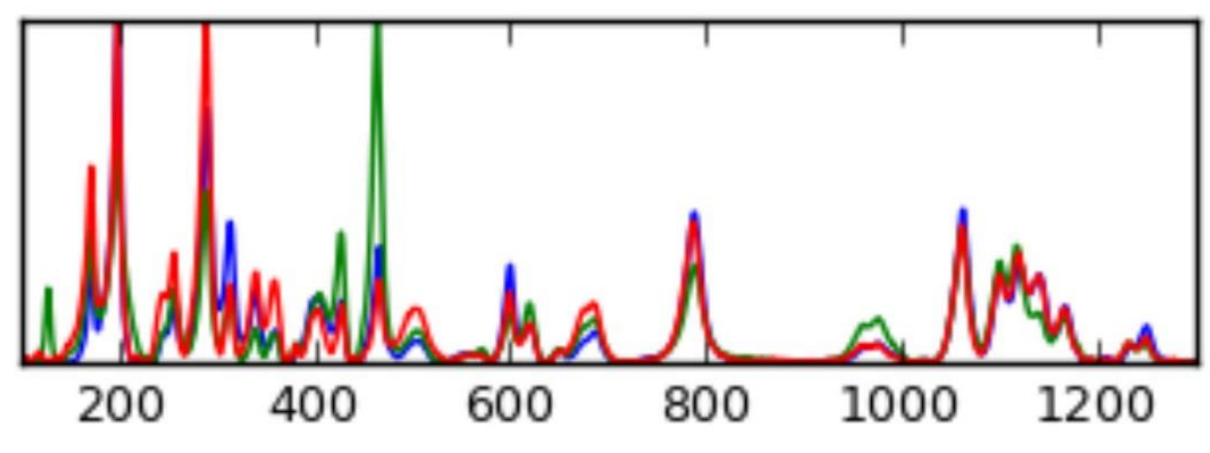

Squashed + Normalized

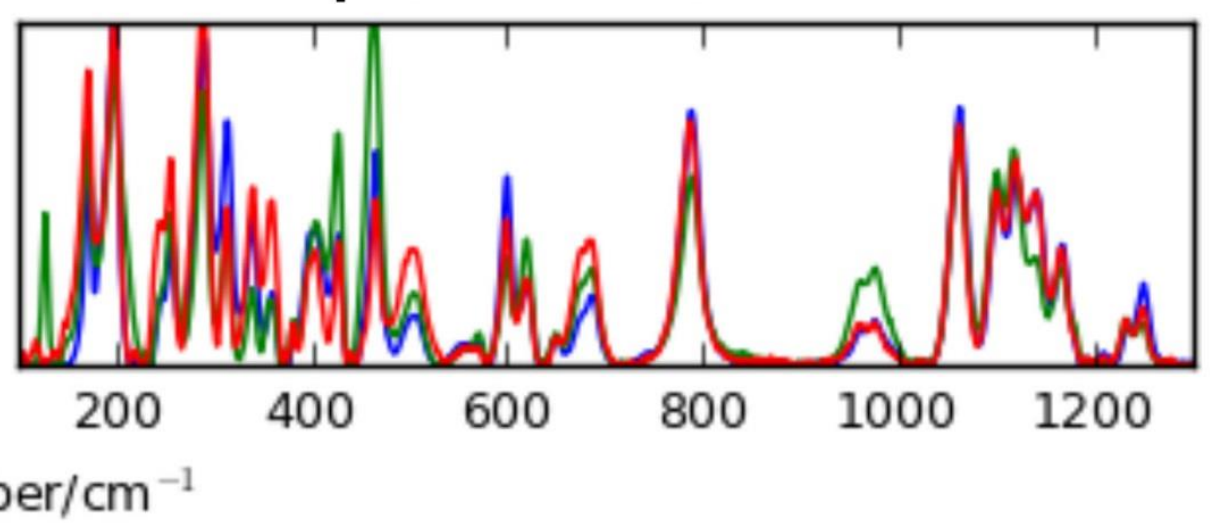

Raman Data

F. Spectral Pre-Processing 
Protocols Based on Individual Peaks and Underlying Physical Principles

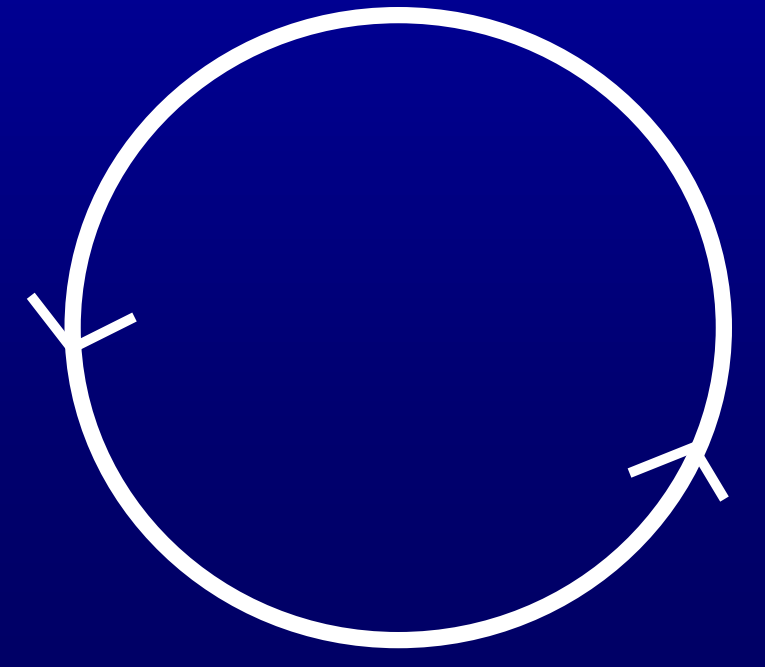

Insights from Machine Learning

Machine Learning can enable fundamental Insights into spectra

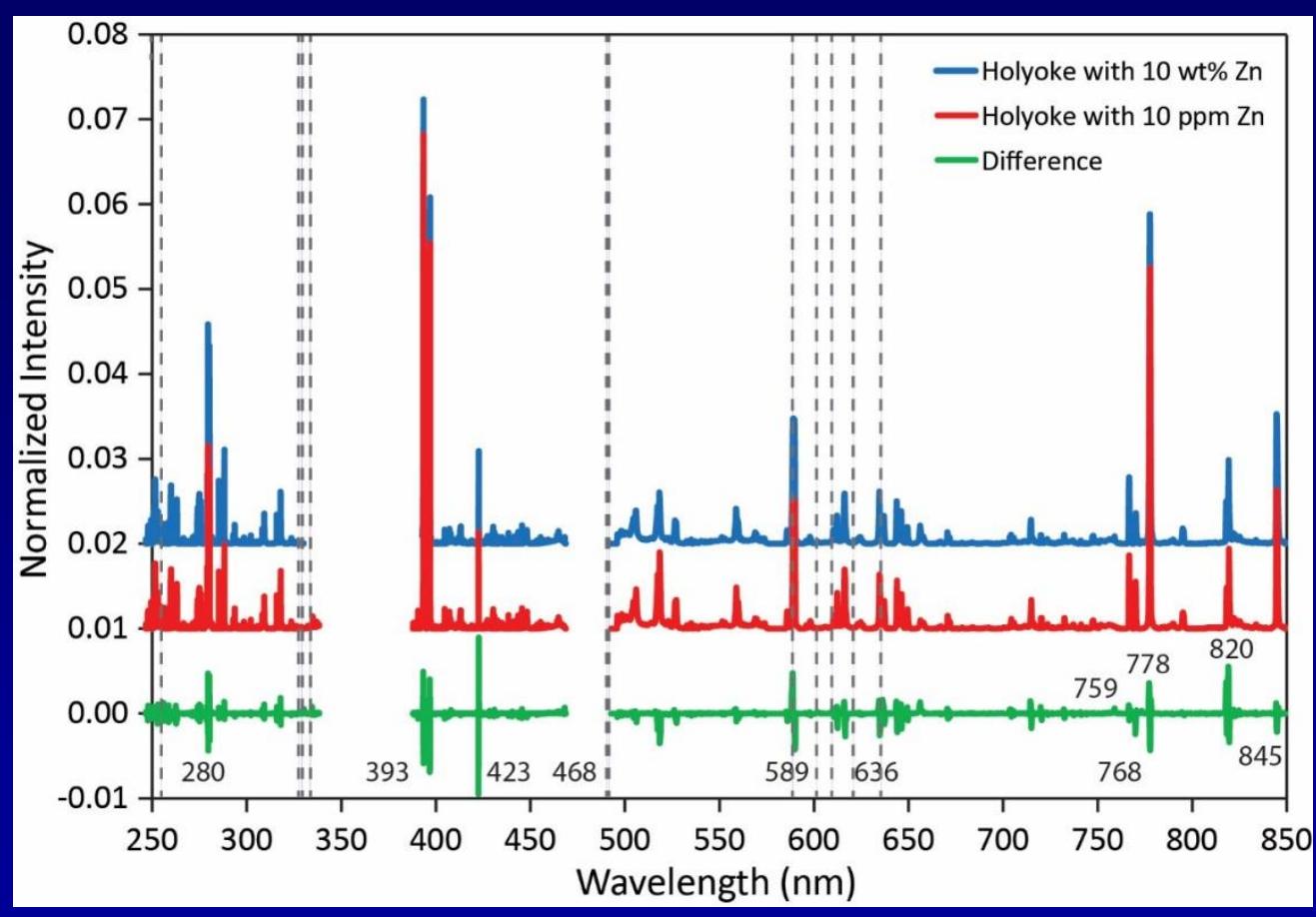




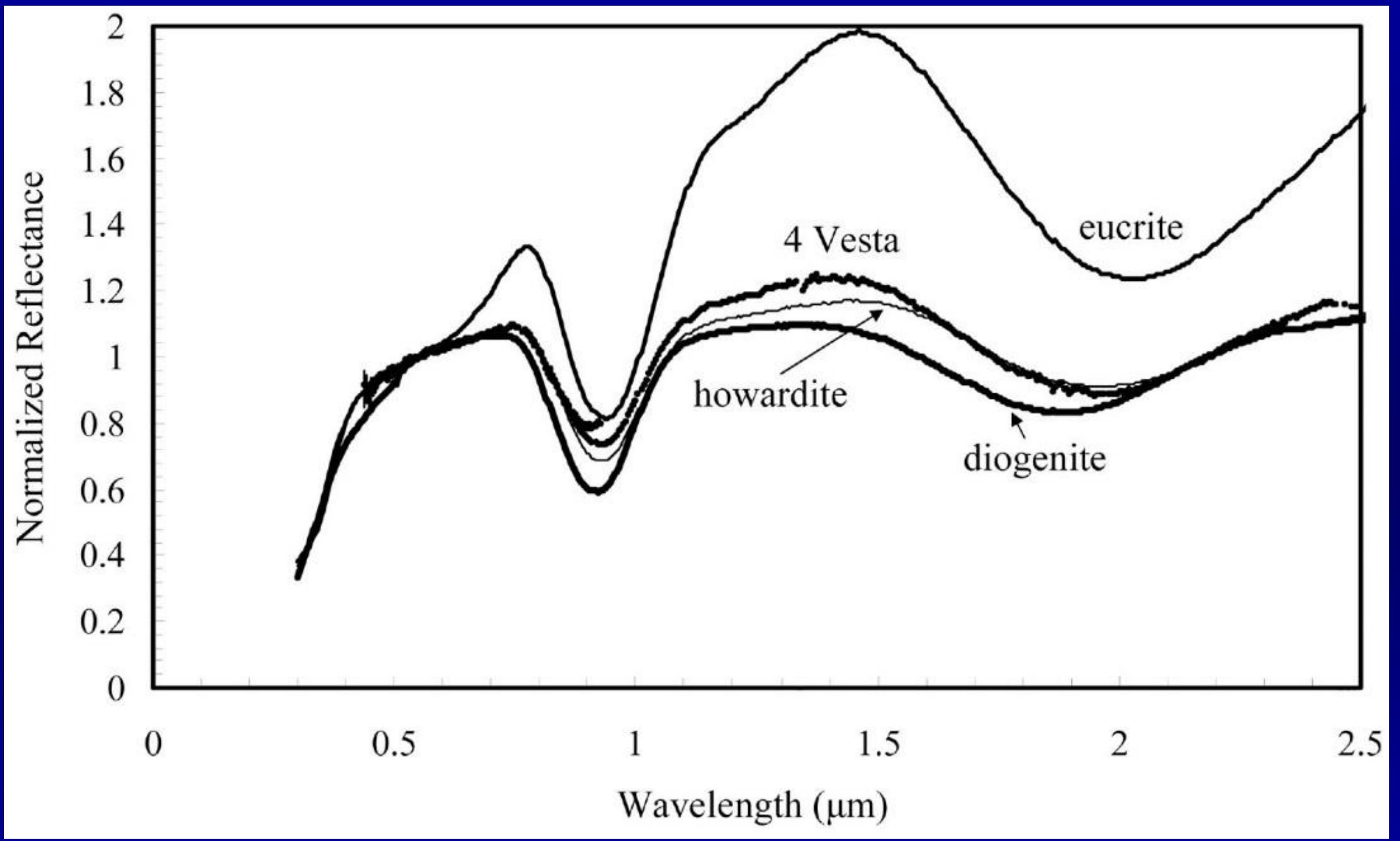

Burbine et al. (2009) 


\section{Barriers to Using Machine Learning}

1. Too little overlap between planetary and computer science communities

2. Steep learning curve to understand new methods

3. Reluctance to move on from fundamentals-based approaches

4. Inadequate and silo-ed spectral databases

5. Ignorance of instrumental differences 


\section{Beneffits of Using Machine Learning}

1. Utilize \& evaluate all channels of spectral data using automated (objective) feature selection

2. Quantifiable error bars for conclusions based on spectral data

3. Improved instrument design for planetary exploration

4. Calibration transfer between data sets

5. Ability to integrate data from multiple types of spectroscopy in a single model

6. Gain new insights into fundamental physical processes 


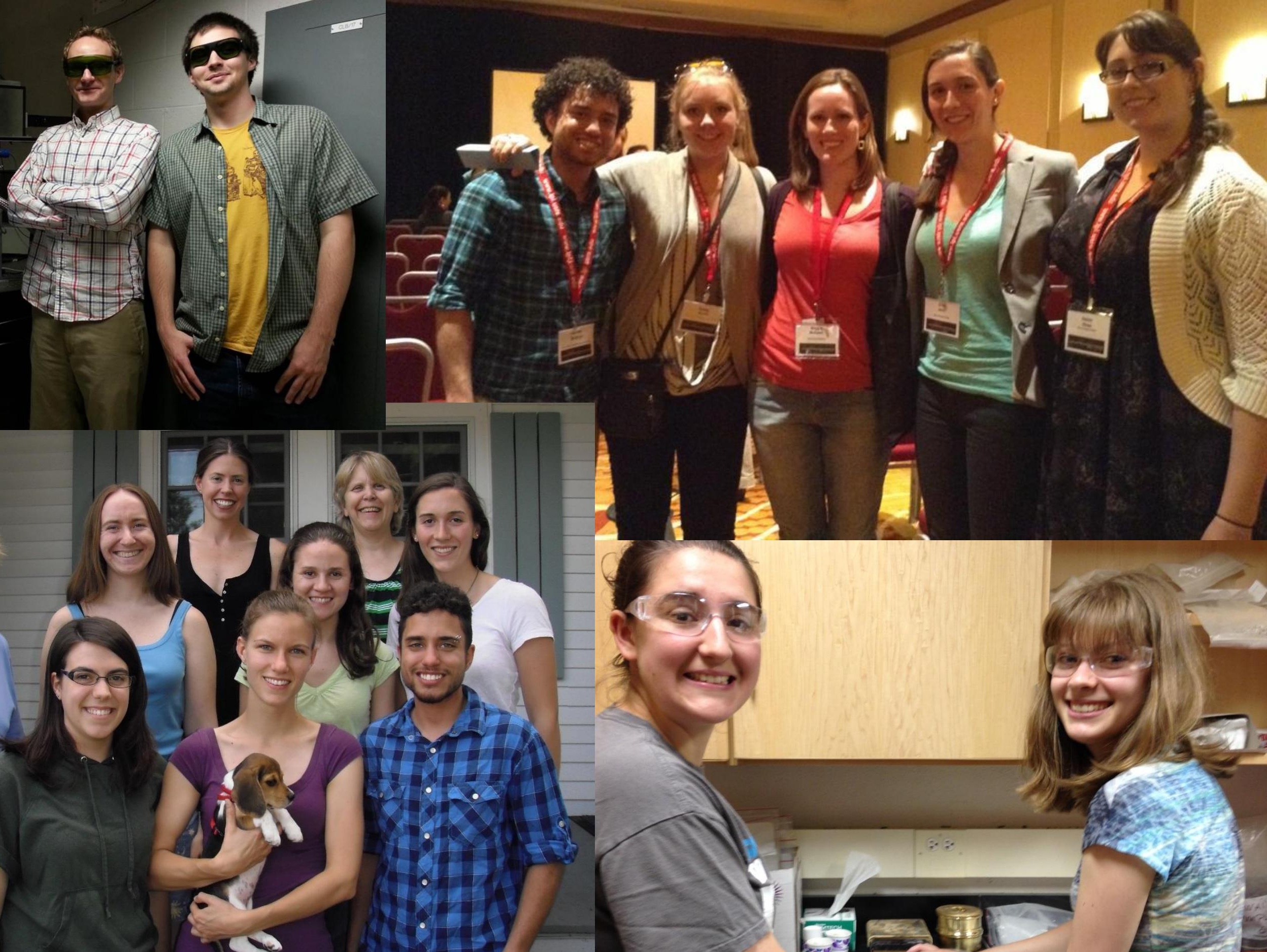




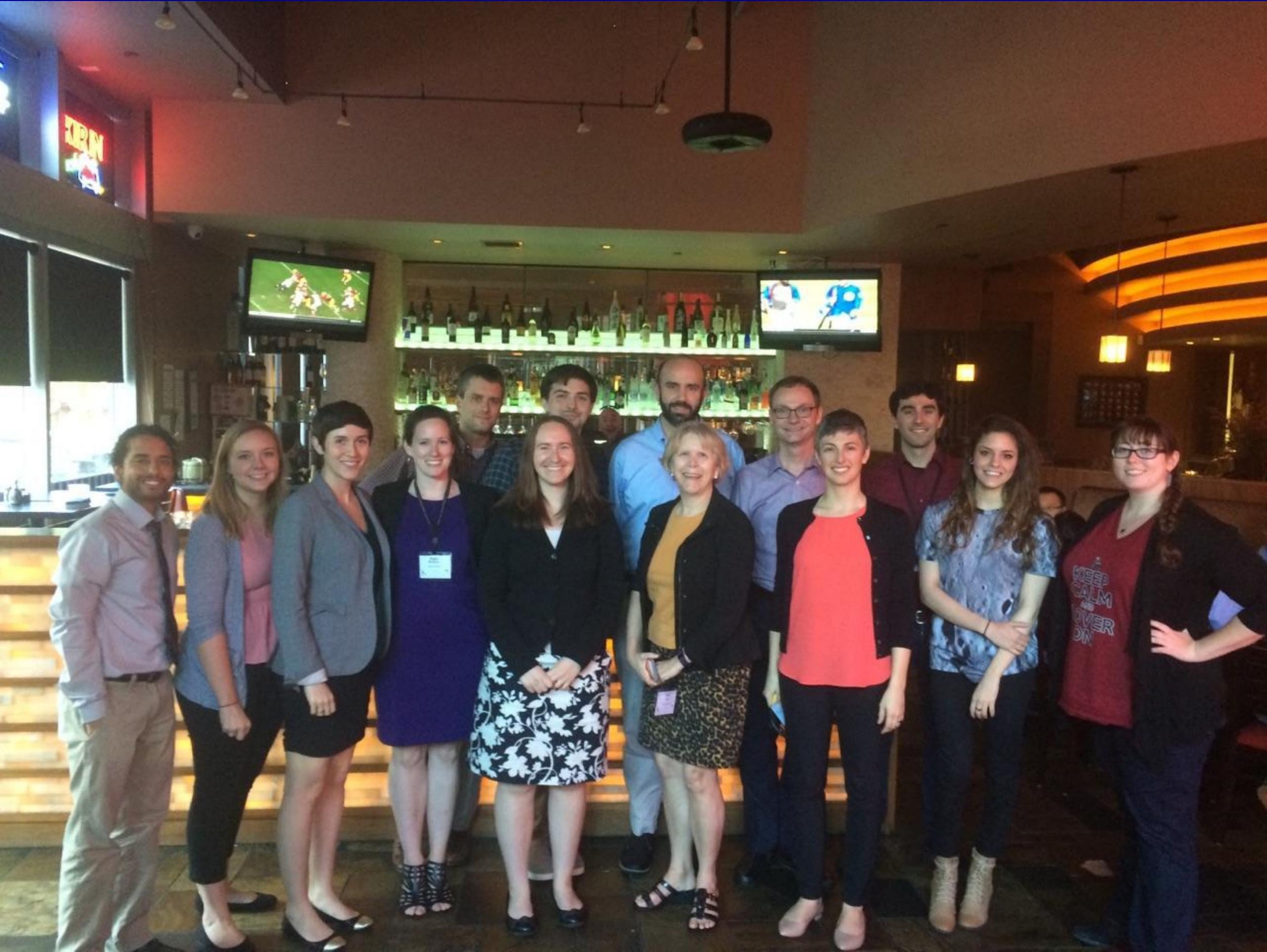

